\title{
Nuclear Reactions of Astrophysical Interest
}

\author{
Pierre Descouvemont* \\ Physique Nucléaire Théorique et Physique Mathématique, Université Libre de Bruxelles, Brussels, Belgium
}

We present different reaction models commonly used in nuclear astrophysics, in particular for the nucleosynthesis of the light elements. Nuclear reactions involved in stellar evolution generally occur at energies much lower than the Coulomb barrier. This property makes the cross sections extremely small, and virtually impossible to be measured in the laboratory. We start with a general discussion of low-energy scattering, and define the various cross sections required for reaction networks (essentially radiative capture and transfer reactions). Then we present specific models. Microscopic theories are based on fundamental principles, such as a nucleon-nucleon interaction, and an exact account of the antisymmetrization between all nucleons. In this context, most calculations performed so far have been done in the cluster approximation, but recent works, referred to as "ab initio" models, go beyond this approximation. Microscopic models can be simplified by neglecting the internal structure of the colliding nuclei, which

OPEN ACCESS

Edited by:

Rosario Gianluca Pizzone, Laboratori Nazionali del Sud (INFN),

Italy

Reviewed by:

Stefan Typel,

Technische Universität Darmstadt,

Germany

Marco La Cognata,

Laboratori Nazionali del Sud (INFN),

\section{Italy}

${ }^{*}$ Correspondence:

Pierre Descouvemont pdesc@ulb.ac.be

Specialty section: This article was submitted to Nuclear Physics,

a section of the journal Frontiers in Astronomy and Space Sciences

Received: 16 January 2020 Accepted: 10 March 2020 Published: 24 April 2020

Citation:

Descouvemont P (2020) Nuclear Reactions of Astrophysical Interest.

Front. Astron. Space Sci. 7:9.

doi: 10.3389/fspas.2020.00009 leads to the potential model, also named the optical model. An alternative approach for the theoretical analysis of the experimental data is based on the phenomenological R-matrix theory, where parameters are fitted to the existing data, and then used to extrapolate the cross sections down to stellar energies. Indirect approaches, such as the Trojan Horse method, are briefly outlined. Finally, we present some typical applications of the different models.

Keywords: nuclear reactions, nuclear astrophysics, scattering models, capture reactions, transfer reactions, indirect methods

\section{INTRODUCTION}

Nuclear reactions are responsible for the nucleosynthesis, i.e., for the formation of the elements in the Universe (Bethe, 1939; Burbidge et al., 1957). Big-Bang nucleosynthesis mainly produces ${ }^{2} \mathrm{H},{ }^{3} \mathrm{He}$, and $\alpha$ particles. This primordial nucleosynthesis is followed by the formation of early stars where elements up to $\mathrm{Fe}$ are produced. Heavier elements are then synthesized by various processes, such as neutron capture (Reifarth et al., 2018), neutrino-induced reactions (AlvarezRuso et al., 2018), explosive events in supernovae (Wiescher et al., 2012), and the rapid-neutron process in neutron-star mergers (Thielemann et al., 2017). Astrophysical scenarios and stellar models are discussed in many textbooks (see e.g., Clayton, 1983; Rolfs and Rodney, 1988; Iliadis, 2007; Thompson and Nunes, 2009) and review articles (see e.g., Aprahamian et al., 2005; José and Iliadis, 2011; Wiescher et al., 2012).

The role of nuclear physics in astrophysics is fundamental, and this discipline is referred to as nuclear astrophysics. It essentially started with the pioneering work of Bethe (1939). Later, the various cycles of stellar evolution were described in the seminal review by Burbidge et al., known as $\mathrm{B}^{2} \mathrm{FH}$ (Burbidge et al., 1957). Many observational properties find their origin in nuclear physics. (i) A typical example is the $0_{2}^{+}$level of ${ }^{12} \mathrm{C}$, known as the Hoyle state (Hoyle, 1954), which was predicted from the observed ${ }^{12} \mathrm{C}$ abundance in the Universe, and found 
experimentally later. The formation of ${ }^{12} \mathrm{C}$ is currently wellunderstood from the triple $\alpha$ process. (ii) From the observed abundances of the elements, a gap between masses 5 and 8 is explained by the particle instability of ${ }^{5} \mathrm{He}$ and ${ }^{5} \mathrm{Li}$. (iii) In the high-mass region of the abundance distribution, peaks are clearly observed, and are explained by the existence of magic numbers in nuclear physics. Magic nuclei are strongly bound, and therefore difficult to destroy by photodissociation. (iv) The abundance distribution also presents an "even-odd" effect, even nuclei being more abundant; again the origin of this effect stems from nuclear binding energies since odd-mass nuclei are less bound and therefore more fragile than even-mass nuclei. Recent reviews on nuclear astrophysics can be found in Bertulani and Kajino (2016) and Liccardo et al. (2018).

Stellar models require many nuclear inputs. A huge number (up to several thousands) of reaction rates, involving charged particles (protons and alphas) and neutrons, are needed in nucleosynthesis networks (Wiescher et al., 2012). Reactions involving charged particles are transfer and capture processes. The former stem from the nuclear force, and the latter from the electromagnetic interaction. When both channels are open, the transfer cross section is always much larger than the capture cross section. A challenge for nuclear physicists is to determine the cross sections at stellar energies, which are in general much lower than the Coulomb barrier. Except in a few cases, direct measurements in this low-energy range (referred to as the "Gamow peak") are not possible, since the cross sections are too low to be measured in the laboratory. Although experimental techniques have been strongly developed over the last decades (Broggini et al., 2010), a theoretical support is often necessary to complement the data, and in particular to extrapolate them down to stellar energies.

Owing to the experimental difficulties associated with direct measurements, several indirect techniques have been developed: the Trojan Horse method (Baur, 1986; Tumino et al., 2013; Spitaleri et al., 2019), the Coulomb breakup method (Baur et al., 1986), or the Asymptotic Normalization Coefficient method (ANC, see Mukhamedzhanov et al., 2001). The main advantage of these indirect approaches is to circumvent the smallness of the cross sections. However, they require a precise theoretical modeling to determine the relevant cross sections from the data.

The calculation of the cross sections is of course based on the scattering theory. Various models are being used in the literature. Owing to the low energies relevant in nuclear astrophysics, and to the low level densities, the optical model (also referred to as "potential model") can be used for capture reactions (Tombrello and Parker, 1963; Bertulani, 2003). Heavy-ion fusion reactions are also described by this model, even if different variants exist (Canto et al., 2006). Impressive developments have been performed in the framework of microscopic models, which present an important predictive power since they rely on a nucleon-nucleon interaction only. Solving a many-body Schrödinger equation for scattering states is however a difficult task, and the cluster approximation (Horiuchi et al., 2012) is used in most calculations. However, recent works succeeded to address, in a microscopic theory, the ${ }^{2} \mathrm{H}(\mathrm{d}, \gamma)^{4} \mathrm{He}$ (Arai et al., 2011), ${ }^{3} \mathrm{He}(\mathrm{d}, \mathrm{p})^{4} \mathrm{He}$ (Navrátil and Quaglioni, 2012), and ${ }^{3} \mathrm{He}(\alpha, \gamma){ }^{7} \mathrm{Be}$ (Neff, 2011; Dohet-Eraly et al., 2016) reactions without the cluster approximation. Recent progress has been made in Effective Field Theories (Zhang et al., 2018). These calculations are highly computer demanding, and are currently limited to low-mass systems.

The models discussed above are, in principle, independent of experimental data. In practice, however, available data are used to assess their reliability, and/or to tune some important parameter(s). In contrast, the phenomenological $R$-matrix theory (see for example Barker and Kajino, 1991; Descouvemont and Baye, 2010) explicitly relies on the existence of data, but is an efficient tool to analyse reactions of astrophysical interest. The cross sections can be parameterized by a small number of real, energy-independent, parameters. This fitting procedure requires the availability of experimental data, but in general allows a reliable extrapolation down to stellar energies. The $R$-matrix theory deals with capture, transfer and elastic scattering on an equal footing. In practice, however, the $R$-matrix theory is limited to reactions where the level density is limited (typically up to a few levels per $\mathrm{MeV}$ ).

When the mass increases, the level density is in general too large for cluster models or for an $R$-matrix approach. In that situation, the cross section essentially depends on properties of the compound nucleus. Here the shell model (Richter et al., 2011) provides information on resonance properties, such as energies, spins, widths, etc. An extension to the continuum has been developed (Chatterjee et al., 2006). The Hauser-Feshbach formalism (Rauscher et al., 1997) is also widely used in high-mass systems, with a high level density.

A specificity of nuclear astrophysics is to require a large number of reaction rates. These reaction rates are obtained from various sources, experimental as well as theoretical. A link between nuclear physics and astrophysics can be established by compilations, where the authors provide an evaluation of the available data, and recommend reaction rates. The first compilations were performed by the Caltech group (Caughlan and Fowler, 1988), and then improved in various ways: evaluation of uncertainties, improved numerical treatment, update of experimental data, etc. Some compilations address specific reactions, such as Big-Bang nucleosynthesis (Descouvemont et al., 2004; Coc et al., 2012) or solar fusion reactions (Adelberger et al., 2011), but other works cover a wider range (Angulo et al., 1999; Longland et al., 2010).

In this review, we discuss the current status of reaction models in nuclear astrophysics. We focus on charged-particle induced reactions, which represent one of the main inputs in stellar evolution. In section 2, we present an overview of the different cross sections involved in the nucleosynthesis. In section 3 we briefly describe some theoretical models used for nuclear reactions. Applications are presented in section 4. The conclusion and outlook are presented in section 5 .

\section{CROSS SECTIONS FOR NUCLEAR ASTROPHYSICS}

\subsection{Scattering Wave Functions}

In this section, we present an outline of the reaction theory needed for nuclear astrophysics. In particular, we are dealing 
with low energies, around and below the Coulomb barrier. The goal is to model different processes, such as transfer or capture reactions. We start from a general formalism of scattering theory, and then apply it to different models. This outline is of course very brief, and we refer to textbooks (e.g., Satchler, 1983; Canto and Hussein, 2013) for a more detailed presentation.

The main goal of scattering models is to solve the Schrödinger equation

$$
H \Psi=E \Psi
$$

for positive energies $E$ (this energy is defined from the reaction threshold). In this equation, $H$ is the Hamiltonian, and $\Psi$ is the nucleus-nucleus wave function. For the sake of simplicity we assume here that the internal structure of the colliding nuclei is neglected and that the spin of the target is zero. Consequently, the Hamiltonian only depends on the relative coordinate $r$. For a system with charges $\left(Z_{1} e, Z_{2} e\right)$ and nucleon numbers $\left(A_{1}, A_{2}\right)$, it is written as

$$
H=-\frac{\hbar^{2}}{2 \mu m_{N}} \Delta_{r}+V_{N}(r)+V_{C}(r),
$$

where, $m_{N}$ is the nucleon mass and $\mu=A_{1} A_{2} /\left(A_{1}+A_{2}\right)$ is the dimensionless reduced mass of the system. In Equation (2), $V_{N}(r)$ and $V_{C}(r)$ are the nuclear and Coulomb potentials, respectively. In general $V_{N}(r)$ depends on the angular momentum and on the spin. A partial wave with total spin $J$ and angular momentum $L$ is given by

$$
\Psi_{L}^{J M}(\boldsymbol{r})=\frac{1}{r} g_{L}^{J}(r)\left[Y_{L}\left(\Omega_{r}\right) \otimes \chi_{s}\right]^{J M},
$$

where the spinor $\chi_{s}$ is associated with the spin. In most cases, $s=0$ or $s=1 / 2$ (the latter case covers the frequent situation of nucleon scattering). The relative function $g_{L}^{J}(r)$ is given by the one-dimension Schrödinger equation

$$
-\frac{\hbar^{2}}{2 \mu m_{N}}\left(\frac{d^{2}}{d r^{2}}-\frac{L(L+1)}{r^{2}}+V_{N}(r)+V_{C}(r)\right) g_{L}^{J}(r)=E g_{L}^{J}(r),
$$

and must be solved for scattering states $(E>0)$ or for bound states $(E<0)$ with the corresponding boundary conditions. Efficient techniques are based on the Numerov algorithm (Raynal, 1972) or on the $R$-matrix method (Descouvemont and Baye, 2010).

At large distances, the nuclear potential $V_{N}$ in (2) is negligible. For a scattering state, the radial wave function tends to

$$
g_{L}^{J}(r) \underset{r \rightarrow \infty}{\longrightarrow} I_{L}(k r)-U_{L}^{J} O_{L}(k r)
$$

where $I_{L}(x)$ and $O_{L}(x)$ are the incoming and outgoing Coulomb functions (see section 2.2), and $U_{L}^{J}$ is the scattering matrix (in single-channel calculations, it is a $1 \times 1$ matrix). The scattering matrix depends on the nuclear potential, and provides the elastic cross sections. For real potentials, usually used in astrophysics, we have the property

$$
\left|U_{L}^{J}\right|=1
$$

The phase shift $\delta_{L}^{J}$ is defined from

$$
U_{L}^{J}=\exp \left(2 i \delta_{L}^{J}\right)
$$

and is real. At energies above the Coulomb barrier, complex potentials are often used. The imaginary part simulates absorption, and the model is referred to as the "optical model" (Satchler, 1983). In that case, we have

$$
\left|U_{L}^{J}\right| \leq 1
$$

and the phase shift contains an imaginary part.

Generalizations can be performed in various directions. A first possibility is to extend the wave function (3) to multichannel calculations as

$$
\Psi_{L}^{J M}(\boldsymbol{r})=\frac{1}{r} \sum_{c L I} g_{c L I}^{J}(r)\left[Y_{L}\left(\Omega_{r}\right) \otimes\left[\phi_{c}^{I_{1}} \otimes \phi_{c}^{I_{2}}\right]^{I}\right]^{J M},
$$

where $\phi_{c}^{I_{1}}$ and $\phi_{c}^{I_{2}}$ are the internal wave functions with spins $I_{1}$ and $I_{2}$, and where $I$ is the channel spin. Equation (4) is replaced by a system of coupled equations.

In Equations (3) and (9), antisymmetrization effects between the target and the projectile are neglected. However, they can be partly simulated by an appropriate choice of the nucleusnucleus potential (Friedrich, 1981; Baye, 1987). A second generalization aims to explicitly include antisymmetrization effects. In microscopic cluster models (Descouvemont and Dufour, 2012), the wave function is defined as

$$
\Psi_{L}^{J M}=\mathcal{A} \frac{1}{r} \sum_{c L I} g_{c L I}^{J}(r)\left[Y_{L}\left(\Omega_{r}\right) \otimes\left[\phi_{c}^{I_{1}}\left(\boldsymbol{\xi}_{1}\right) \otimes \phi_{c}^{I_{2}}\left(\boldsymbol{\xi}_{2}\right)\right]^{I}\right]^{J M},
$$

where $\mathcal{A}$ is the $A$-nucleon antisymmetrization operator. The internal wave functions are defined in the shell model and depend on a set of internal coordinates $\left(\boldsymbol{\xi}_{\boldsymbol{i}}\right)$. We will briefly describe microscopic models in section 3 .

\subsection{General Properties of Low-Energy Reactions}

Before discussing applications specific to nuclear astrophysics, we address here some general properties of the cross sections at low energies, typical of stellar conditions. In this energy regime, the scattering between charged particles is essentially governed by the Coulomb interaction. In other words, we can neglect the structure of the nuclei, and reduce the Schrödinger equation to a two-body problem. For a given angular momentum $L$, the wave function depends on the relative coordinate $r$. The radial Schrödinger equation only involves the Coulomb potential and reads, at c.m. energy $E$,

$$
-\frac{\hbar^{2}}{2 \mu m_{N}}\left(\frac{d^{2}}{d r^{2}}-\frac{L(L+1)}{r^{2}}\right) g_{L}(r)+\frac{Z_{1} Z_{2} e^{2}}{r} g_{L}(r)=E g_{L}(r)
$$

where we have dropped index $J$. The solutions of Equation (11) are the regular and irregular Coulomb functions $F_{L}(\eta, k r)$ and 
$G_{L}(\eta, k r)$ (Thompson, 2010). They depend on the wave number $k$, and on the Sommerfeld parameter $\eta$

$$
\begin{aligned}
& k=\sqrt{\frac{2 \mu m_{N} E}{\hbar^{2}}} \\
& \eta=\frac{Z_{1} Z_{2} e^{2}}{\hbar v} \approx 0.158 Z_{1} Z_{2} \sqrt{\frac{\mu}{E}}(E \text { in } \mathrm{MeV}),
\end{aligned}
$$

where $v=\hbar k / \mu m_{N}$ is the relative velocity.

From the Coulomb functions, one defines the incoming and outgoing functions as

$$
\begin{aligned}
& I_{L}(\eta, x)=G_{L}(\eta, x)-i F_{L}(\eta, x), \\
& O_{L}(\eta, x)=G_{L}(\eta, x)+i F_{L}(\eta, x) .
\end{aligned}
$$

The penetration factor $P_{L}$ and the shift factor $S_{L}$ are obtained, at a radius $a$, from

$$
\begin{aligned}
& P_{L}(E, a)=k a /\left[F_{L}^{2}(\eta, k a)+G_{L}^{2}(\eta, k a)\right] \\
& S_{L}(E, a)=\left[F_{L}(\eta, k a) F_{L}^{\prime}(\eta, k a)+G_{L}(\eta, k a) G_{L}^{\prime}(\eta, k a)\right] P_{L}(E, a),
\end{aligned}
$$

where the prime denotes the derivative with respect to $k a$. The penetration factor can be approximately interpreted as the probability to cross the Coulomb barrier, and therefore strongly depends on energy (strictly speaking, however, $P_{L}$ is not a probability since it may be larger than unity). Below the Coulomb barrier, it essentially depends on energy as

$$
P_{L}(E, a) \sim \exp (-2 \pi \eta)
$$

This term represents the main contribution to the energy dependence for $L=0$. Higher-order corrections can be found in Clayton (1983) and Afanasjev et al. (2012). The penetration factor is illustrated in Figure $\mathbf{1}$ for a light system $\mathrm{p}+{ }^{12} \mathrm{C}$, and for an heavier system $\alpha+{ }^{12} \mathrm{C}$. The Coulomb barriers are around 1.6 and $3.2 \mathrm{MeV}$, respectively. As expected, the centrifugal barrier reduces the penetration factor. This effect is stronger for light systems, where the reduced mass is small. Some dependence upon the radius $a$ shows up, but is weak at very low energies.

The fast energy dependence (15) is common to all low-energy cross sections. For this reason, nuclear astrophysicists use the $S$ factor

$$
S(E)=\sigma(E) E \exp (2 \pi \eta)
$$

which presents a smooth energy dependence for non-resonant reactions. It contains the nuclear information on the reaction.

Resonances play an important role in many reactions. Near a resonance energy $E_{R}$ in a partial wave $J_{R}$, a cross section $\sigma(E)$ can be written at the Breit-Wigner approximation as

$$
\sigma(E) \approx \frac{\pi}{k^{2}} \frac{2 J_{R}+1}{\left(2 I_{1}+1\right)\left(2 I_{2}+1\right)} \frac{\Gamma_{i}(E) \Gamma_{f}(E)}{\left(E-E_{R}\right)^{2}+\Gamma(E)^{2} / 4},
$$

where $\Gamma_{i}$ and $\Gamma_{f}$ are the partial widths in the initial and final channels, $\Gamma$ is the total width, and $\left(I_{1}, I_{2}\right)$ are the spins of the colliding nuclei. This definition is valid for transfer as well as for capture reactions. In both cases, the entrance width $\Gamma_{i}(E)$ is a particle width, often a proton or an alpha width. Its energy dependence is given by

$$
\Gamma_{i}(E)=\Gamma_{i} \frac{P_{L}(E, a)}{P_{L}\left(E_{R}, a\right)}=2 \gamma_{i}^{2} P_{L}(E, a),
$$

where $\Gamma_{i}$ is the width at energy $E_{R}$, and $\gamma_{i}^{2}$ is called the reduced width (Descouvemont and Baye, 2010). Notice that the energy variation slightly depends on the radius $a$. The Breit-Wigner approximation is a particular case of the more general $R$-matrix theory (Lane and Thomas, 1958; Descouvemont and Baye, 2010), where several resonances may overlap.

According to Equation (15), the width of a resonance gets very narrow when the energy decreases. The reduced width $\gamma_{i}^{2}$ reflects the cluster structure of the resonance (Descouvemont and Baye, 2010) and is defined even for bound states (in that case the total width is $\Gamma_{i}=0$ since the penetration factor vanishes). It does not depend on energy. Consequently, even a state presenting a strong cluster structure may be characterized by a small total width
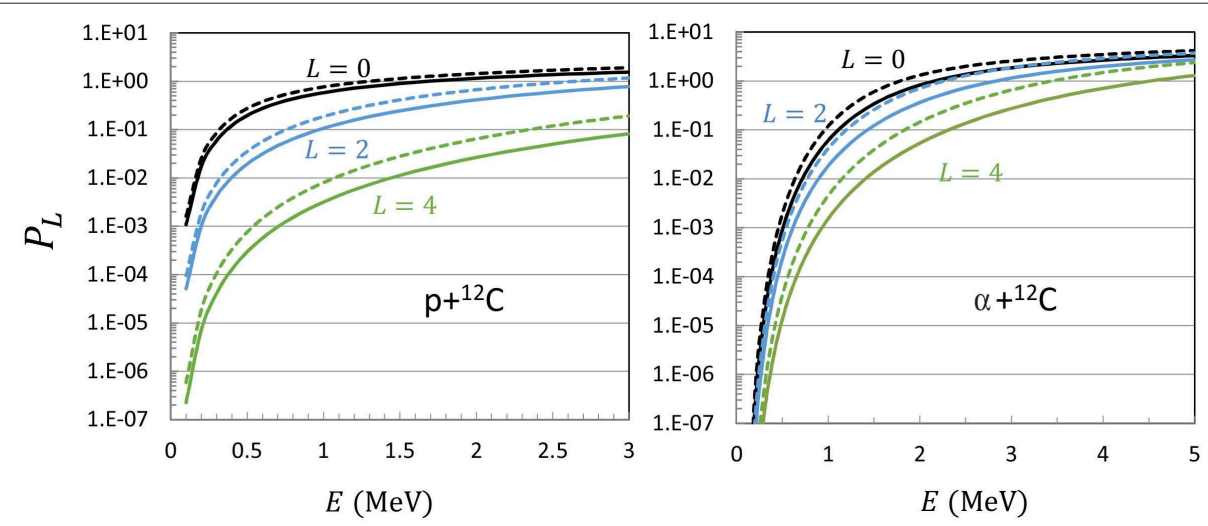

FIGURE 1 | Penetration factor (14) for the $\mathrm{p}+{ }^{12} \mathrm{C}$ (left) and $\alpha+{ }^{12} \mathrm{C}$ (right) systems. Solid and dashed lines correspond to $\mathrm{a}=5 \mathrm{fm}$ and $\mathrm{a}=6 \mathrm{fm}$, respectively. 
if it is located at low energies. As the entrance width depends on the penetration factor, the fast Coulomb dependence is also removed in the resonant $S$ factor [Equation (16) with the cross section (17)].

In transfer reactions, $\Gamma_{f}(E)$ is also a particle width, and the previous discussion is still valid. Notice that, in general, the $L$ values are different (see a discussion and examples in Descouvemont, 2003). However, the $Q$ value must be taken into account as

$$
\Gamma_{f}(E)=2 \gamma_{f}^{2} P_{L}(E+Q, a)
$$

where $\gamma_{f}^{2}$ is the reduced width of the resonance in the exit channel. If the threshold energy $Q$ is large (typically a few $\mathrm{MeV}$ ), the corresponding penetration factor presents a slow energy dependence, and the final width is approximated by a constant. This is not true in a few specific reactions, where the $Q$ value is small (or even negative), and where the energy dependence of (19) is not negligible (an example is ${ }^{17} \mathrm{O}(\alpha, \mathrm{n}){ }^{20} \mathrm{Ne}$ where $Q=$ $0.586 \mathrm{MeV})$.

In capture reactions, the width of the exit channel $\Gamma_{f}$ is the $\gamma$ width. The energy dependence is given by the theory of electromagnetic transitions and reads

$$
\Gamma_{\gamma}(E)=\Gamma_{\gamma}\left(\frac{E-E_{f}}{E_{R}-E_{f}}\right)^{2 \lambda+1},
$$

where $\Gamma_{\gamma}$ is the $\gamma$ width at the resonance energy, $\lambda$ is the order of the electromagnetic transition, and $E_{f}$ the energy of the final nuclear state $\left(E_{f}<0\right)$. In general this energy dependence is rather weak, and can be neglected. Owing to the lower amplitude of the electromagnetic interaction compared to the nuclear force, $\gamma$ widths are in general much lower than particle widths (in light nuclei $\left.\Gamma_{\gamma} \lesssim 1 \mathrm{eV}\right)$.

The total width $\Gamma(E)$ in (17) is in general defined as the sum of the partial widths,

$$
\Gamma(E)=\Gamma_{i}(E)+\Gamma_{f}(E) .
$$

If more than two channels are open, the total width should include all partial widths of the resonance.

\subsection{Radiative-Capture Reactions}

Radiative-capture reactions play an important role in the nucleosynthesis (Iliadis, 2007). They can be seen as a transition from an initial scattering state to a final bound state of the system. This process arises from the electromagnetic interaction, and can therefore be treated in the perturbation theory (Rose and Brink, 1967). If $H_{e}$ is the photon-emission Hamiltonian for the nuclear system, the capture cross section to a final state with spin $J_{f}$ and parity $\pi_{f}$ is given by

$$
\begin{aligned}
\frac{d \sigma_{c}}{d \Omega_{\gamma}}\left(E, J_{f} \pi_{f}\right)= & \frac{k_{\gamma}}{2 \pi \hbar} \frac{1}{\left(2 I_{1}+1\right)\left(2 I_{2}+1\right)} \\
& \times \sum_{q \nu_{1} \nu_{2} M_{f}}\left|\left\langle\Psi^{J_{f} M_{f} \pi_{f}}\left|H_{e}\left(q, \Omega_{\gamma}\right)\right| \Psi^{\nu_{1} \nu_{2}}\right\rangle\right|^{2}
\end{aligned}
$$

where $\Omega_{\gamma}$ is the photon angle, $q= \pm 1$ is the photon polarization, and $\Psi^{J_{f} M_{f} \pi_{f}}$ the final-state wave function. Here $v_{1}$ and $\nu_{2}$ are the spin projections of the colliding nuclei.

To compute the cross section, the initial wave function $\Psi^{v_{1} v_{2}}$ is expanded in partial waves, and the electromagnetic operator is expanded in multipoles $\mathcal{M}_{\lambda \mu}^{\sigma}$ (Rose and Brink, 1967). At the long wavelength approximation, the electric $(\sigma=E)$ and magnetic $(\sigma=M)$ multipole operators are given by

$$
\begin{aligned}
& \mathcal{M}_{\lambda \mu}^{E}=e \sum_{i}^{A} g_{\ell}(i) r_{i}^{\lambda} Y_{\lambda}^{\mu}\left(\Omega_{r_{i}}\right) \\
& \mathcal{M}_{\lambda \mu}^{M}=\mu_{N} \sum_{i}^{A}\left[\frac{2}{\lambda+1} g_{\ell}(i) \boldsymbol{\ell}_{i}+g_{s}(i) \boldsymbol{s}_{i}\right] \cdot \nabla r_{i}^{\lambda} Y_{\lambda}^{\mu}\left(\Omega_{r_{i}}\right),
\end{aligned}
$$

with

$$
\begin{aligned}
& g_{\ell}(i)=1 / 2-t_{i z}, \\
& g_{s}(i)=g_{p}\left(1 / 2-t_{i z}\right)+g_{n}\left(1 / 2+t_{i z}\right),
\end{aligned}
$$

where $t_{i z}$ is the isospin projection of nucleon $i$, and $g_{p}, g_{n}$ are the gyromagnetic factors of the proton $\left(g_{p}=5.586\right)$ and of the neutron $\left(g_{n}=-3.826\right)\left(\mu_{N}=e \hbar / m_{N} c\right.$ is the nuclear magneton). Equations (23) are written in the framework of a microscopic approach. Simplified expressions, valid for two-particle systems can be found (e.g., in Bertulani, 2003; Descouvemont, 2003). After integration over the photon angle $\Omega_{\gamma}$, the total cross section is given by

$$
\begin{aligned}
\sigma_{c}\left(E, J_{f} \pi_{f}\right)= & \frac{2 J_{f}+1}{\left(2 I_{1}+1\right)\left(2 I_{2}+1\right)} \frac{8 \pi}{\hbar} \\
& \times \sum_{\sigma \lambda J_{i} I_{\omega} L_{\omega}} \frac{k_{\gamma}^{2 \lambda+1}}{2 L_{\omega}+1} \frac{(\lambda+1)}{\lambda(2 \lambda+1) ! !^{2}} \\
& \left|\left\langle\Psi^{J_{f} \pi_{f}}|| \mathcal{M}_{\lambda}^{\sigma}|| \Psi_{L_{\omega} I_{\omega}}^{J_{i} \pi_{i}}(E)\right\rangle\right|^{2},
\end{aligned}
$$

where $J_{i}$ and $\pi_{i}$ are the spin and parity in the entrance channel, and where $L_{\omega}$ and $I_{\omega}$ are the channel spin and the orbital momentum. The summations in (25) are limited by the usual selection rules

$$
\begin{aligned}
& \left|J_{i}-J_{f}\right| \leq \lambda \leq J_{i}+J_{f}, \\
& \pi_{i} \pi_{f}=(-)^{\lambda}(\text { for } \sigma=E), \\
& \pi_{i} \pi_{f}=(-)^{\lambda+1}(\text { for } \sigma=M) .
\end{aligned}
$$

In addition, the long-wavelength approximation $\left(k_{\gamma} R \ll 1\right.$, where $R$ is a typical dimension of the system) strongly reduces the summation over $\lambda$. In many cases, a single multipole is important.

As in the previous subsection we only give the integrated cross section, where no interference between multipoles and partial waves shows up. In contrast, the differential cross section (22) involves interference terms (see more detail in Descouvemont, 2003). 


\subsection{Transfer Reactions}

In a transfer reaction (also denoted as "rearrangement reaction"), some nucleons are exchanged between the target and the projectile. A typical example in astrophysics is the ${ }^{13} \mathrm{C}(\alpha, n){ }^{16} \mathrm{O}$ reaction, where two protons and one neutron are stripped from the $\alpha$ particle to ${ }^{13} \mathrm{C}$, to produce ${ }^{16} \mathrm{O}$. Transfer processes arise from the nuclear interaction and the corresponding cross sections are usually much larger than the capture cross sections, arising from the electromagnetic interaction. For example, the ${ }^{13} \mathrm{C}(\alpha, \mathrm{n}){ }^{16} \mathrm{O}$ cross section is larger by several order of magnitude than the ${ }^{13} \mathrm{C}(\alpha, \gamma){ }^{17} \mathrm{O}$ cross section.

If $\Omega=(\theta, \phi)$ is the relative direction of the nuclei in the final channel, the transfer cross section (Descouvemont and Baye, 2010) is given by

$$
\frac{d \sigma_{t}}{d \Omega}(E, i \rightarrow f)=\frac{\pi}{k^{2}} \frac{1}{\left(2 I_{1}+1\right)\left(2 I_{2}+1\right)} \sum_{j} A_{j}(E) P_{j}(\cos \theta),
$$

where the anisotropy coefficients $A_{j}(E)$ are defined as

$$
\begin{aligned}
A_{j}(E)= & \frac{1}{4 \pi} \sum_{J \pi \ell L I} \sum_{J^{\prime} \pi^{\prime} \ell^{\prime} L^{\prime} I^{\prime}}(-)^{I-I^{\prime}} Z\left(j, J, J^{\prime}, \ell, L, I\right) Z\left(j, J, J^{\prime}, \ell^{\prime}, L^{\prime}, I^{\prime}\right) \\
& U_{i \ell I, f \ell^{\prime} I^{\prime}}^{J \pi}(E) U_{i L I, f L^{\prime} I^{\prime}}^{J^{\prime} \pi^{\prime} *}(E) .
\end{aligned}
$$

and $U^{J \pi}$ represents the collision matrix generalized to multichannel systems. Coefficients $Z$ are defined as

$$
\begin{aligned}
Z\left(j, J, J^{\prime}, \ell, L, I\right)= & {\left[(2 J+1)\left(2 J^{\prime}+1\right)(2 \ell+1)(2 L+1)\right]^{1 / 2} } \\
& <\ell 0 L 0 \mid j 0>\left\{\begin{array}{lll}
\ell & L & j \\
J^{\prime} & J & I
\end{array}\right\} .
\end{aligned}
$$

Again, definition (27) is model independent. The choice of the model only affects the collision matrix. As for radiative capture, the important quantity in astrophysics is the integrated cross section

$$
\sigma_{t}(E, i \rightarrow f)=\frac{\pi}{k^{2}} \sum_{J \pi} \frac{2 J+1}{\left(2 I_{1}+1\right)\left(2 I_{2}+1\right)} \sum_{L L^{\prime} I I^{\prime}}\left|U_{i L I, f L^{\prime} I^{\prime}}^{J \pi}(E)\right|^{2} .
$$

The discussion of subsection 2.3 remains valid: in general a few terms are important in (30), since only low $L$ values contribute at energies below the Coulomb barrier.

\subsection{Fusion Reactions}

A number of nuclear reactions that occur in stars are fusion reactions, involving light to medium-mass nuclei. Important examples are the carbon and oxygen burning processes in massive stars. The angular momentum and energy-dependent transmission coefficient $T_{f}(L, E)$ is then used in the cross section formula to obtain the fusion cross section $\sigma_{f}$

$$
\sigma_{f}(E)=\frac{\pi}{k^{2}}\left(1+\delta_{12}\right) \sum_{L}(2 L+1) T_{f}(L, E)
$$

where $\delta_{12}=1$ for symmetric systems and 0 otherwise. For symmetric systems the sum runs over even $L$ values only.
Within an optical model approach to fusion (in that case, $\left.\left|U_{L}^{J}\right|<1\right)$, the transmission coefficient is given by

$$
T_{f}(L, E)=1-\left|U_{L}^{J}\right|^{2}
$$

However, at very low energies, $U_{L}^{J} \approx 1$, and this technique is not accurate. From the continuity equation, the transmission coefficient (32) is strictly equivalent to

$$
T_{f}(L, E)=-\frac{2}{\hbar v} \int\left|g_{L}(r)\right|^{2} W(r) d r,
$$

where $W(r)$ is the imaginary part of the optical potential that represents absorption due to fusion, and $g_{L}(r)$ is the exact (singlechannel) wave function (Hussein, 1984; Canto et al., 2006).

\subsection{Weak-Capture Cross Sections}

For low-mass stars, the nucleosynthesis is initiated by the $\mathrm{p}\left(\mathrm{p}, e^{+} v\right) \mathrm{d}$ reaction, which occurs through the weak interaction. Since the corresponding Hamiltonian is much smaller than the nuclear and electromagnetic Hamiltonians, the cross section is very small. Estimates in optimal experimental conditions (Rolfs and Rodney, 1988) predict one event per $10^{6}$ years! Fortunately, theoretical models are quite accurate (Kamionkowski and Bahcall, 1994; Marcucci et al., 2013). The cross section is shown to be proportional to

$$
\sigma_{p p}(E) \sim\left|<\Psi_{d}^{1^{+}}\left\|\mathcal{M}_{F}+\lambda^{2} \mathcal{M}_{G T}\right\| \Psi_{p p}(E)>\right|^{2},
$$

where $\Psi_{d}^{1^{+}}$is the deuteron wave function, $\Psi_{p p}(E)$ is the $p-p$ scattering wave function and $\mathcal{M}_{F}$ and $\mathcal{M}_{G T}$ are the Fermi and Gamow-Teller operators, respectively. In (34), $\lambda^{2}$ is the ratio of axial-vector to vector coupling constants. A recent calculation (Gaspard et al., 2019) provides $S(0) \approx 3.95 \times 10^{-25} \mathrm{MeV}-\mathrm{b}$, which is considerably lower than values obtained for capture or transfer reactions.

\section{REACTION MODELS IN NUCLEAR ASTROPHYSICS}

\subsection{Microscopic Models}

Microscopic models are based on fundamental principles of quantum mechanics, such as the treatment of all nucleons, with exact antisymmetrization of the wave functions. Neglecting three-body forces, the Hamiltonian of a $A$-nucleon system is written as

$$
H=\sum_{i=1}^{A} T_{i}+\sum_{i<j=1}^{A} V_{i j}
$$

where $T_{i}$ is the kinetic energy and $V_{i j}$ a nucleon-nucleon interaction (Wildermuth and Tang, 1977).

The Schrödinger equation associated with this Hamiltonian cannot be solved exactly when $A>3$. For very light systems ( $A \sim 4-5$ ) efficient methods (Kievsky et al., 2008) exist, even for continuum states (Navrátil et al., 2010). However, most 
reactions relevant in nuclear astrophysics involve heavier nuclei, essentially with nucleon or $\alpha$ projectiles. Recent developments of $a b$ initio models (see e.g., Caurier and Navrátil, 2006; Navrátil and Quaglioni, 2011) are quite successful for spectroscopic properties of low-lying states. These models make use of realistic interactions, including three-body forces, and fitted on many properties of the nucleon-nucleon system. Recent works succeeded in applying $a b$ initio calculations to nuclear astrophysics (see e.g., Arai et al., 2011; Neff, 2011; Dohet-Eraly et al., 2016; Marcucci, 2018). However, a consistent description of bound and scattering states of an $A$-body problem remains a challenging task (Navrátil et al., 2010), in particular for transfer reactions.

In the cluster approximation, it is assumed that the nucleons are grouped in clusters (Wildermuth and Tang, 1977; Descouvemont and Dufour, 2012). The internal wave functions of the clusters are denoted as $\phi_{i}^{I_{i} \pi_{i} \nu_{i}}\left(\xi_{i}\right)$, where $I_{i}$ and $\pi_{i}$ are the spin and parity of cluster $i$, and $\xi_{i}$ represents a set of their internal coordinates. In a two-cluster system, a channel function is defined as

$$
\varphi_{L I}^{J M \pi}\left(\Omega_{r}, \boldsymbol{\xi}_{\mathbf{1}}, \boldsymbol{\xi}_{\mathbf{2}}\right)=\left[Y_{L}\left(\Omega_{r}\right) \otimes\left[\phi_{1}^{I_{1} \pi_{1}}\left(\boldsymbol{\xi}_{\mathbf{1}}\right) \otimes \phi_{2}^{I_{2} \pi_{2}}\left(\boldsymbol{\xi}_{\mathbf{2}}\right)\right]^{I}\right]^{J M},
$$

where different quantum numbers show up: the channel spin $I$, the relative angular momentum $L$, the total spin $J$ and the total parity $\pi=\pi_{1} \pi_{2}(-)^{L}$.

As discussed in section 2.1, the total wave function of the $A$-nucleon system is written, in a microscopic cluster model, as

$$
\Psi^{J M \pi}=\sum_{c L I} \Psi_{c L I}^{J M \pi}=\sum_{c L I} \mathcal{A} g_{c L I}^{J \pi}(r) \varphi_{c L I}^{J M \pi}\left(\Omega_{r}, \boldsymbol{\xi}_{\mathbf{1}}, \boldsymbol{\xi}_{2}\right),
$$

which corresponds to the Resonating Group (RGM) definition (Horiuchi, 1977; Descouvemont and Dufour, 2012). Index $c$ refers to different two-cluster arrangements, and $\mathcal{A}$ is the antisymmetrization operator. In most applications, the internal cluster wave functions $\phi_{i}^{I_{i} \pi_{i} v_{i}}$ are defined in the shell model. Accordingly, the nucleon-nucleon interaction must be adapted to this choice, which leads to effective forces, such as the Volkov (Volkov, 1965) or the Minnesota (Thompson et al., 1977) interactions. The relative wave functions $g_{c L I}^{J \pi}(r)$ are to be determined from the Schrödinger equation, which is transformed into a integro-differential equation involving a nonlocal potential (Horiuchi, 1977). In practice, this relative function is expanded over Gaussian functions (Wildermuth and Tang, 1977; Dufour and Descouvemont, 2011), which corresponds to the Generator Coordinate Method (GCM). The numerical calculations can be made systematic with the GCM, which is not the case for the RGM.

The main advantage of cluster models with respect to other microscopic theories is their ability to deal with reactions, as well as with nuclear spectroscopy. The first applications were done for reactions involving light nuclei, such as $\mathrm{d},{ }^{3} \mathrm{He}$ or $\alpha$ particles (Liu et al., 1981; Hofmann and Hale, 1997). More recently, much work has been devoted to the improvement of the internal wave functions: multicluster descriptions (Descouvemont and Baye, 1994), large-basis shell model extensions (Descouvemont, 1996), or monopolar distortion (Baye and Kruglanski, 1992).

\subsection{The Potential Model}

Solving the Schrödinger equation associated with a many-body system is a difficult problem, which does not have an exact solution when the nucleon number is larger than three. The potential model is fairly simple to use, and has been applied to several reactions in low-energy nuclear physics (Tombrello, 1965; Bertulani, 2003). The basic assumptions of the potential model are: (i) the nucleon-nucleon interaction is replaced by a nucleusnucleus force $V(r)$, which depends on the relative coordinate $r$ only; (ii) the wave functions of the unified nucleus can be described by a cluster structure with $A_{1}+A_{2}$ nucleons; (iii) the internal structure of the nuclei does not play any role. Since we are dealing with low energies, the potential is in general real. The extension to higher energies, which requires complex potentials to simulate absorption channels, is known as the optical model. A generalization to coupled-channel problems is also possible, but seldom used in nuclear astrophysics.

The radial function $g_{L}^{J \pi}(r)$ for bound and scattering states is deduced from Equation (4). According to the application, the choice of the nuclear contribution is guided by experimental constraints. In radiative-capture calculations it is crucial to reproduce the final-state energy. If phase shifts are available, they can be used to determine the initial potential.

Besides experimental constraints, the nucleus-nucleus potential must follow requirements arising from microscopic arguments (Kukulin et al., 1983; Baye and Descouvemont, 1985). In the microscopic wave function (37), it can be shown that, under some assumptions, there are non-vanishing radial functions $g_{c L I}^{J \pi}(r)$ which yield $\Psi_{c L I}^{J M \pi}=0$ after application of the antisymmetrization operator $\mathcal{A}$. These radial functions are called "forbidden states," and their number depends on the system and on the angular momentum. To illustrate the problem, let us consider the $\alpha+\mathrm{p}$ system where the $\alpha$ particle is described in the shell model. Since the $s$-shell is filled by the $\alpha$ orbitals, the external proton cannot occupy this $s$-shell state, which corresponds to a forbidden state. We refer the reader to Buck et al. (1977), Kukulin et al. (1983), and Baye and Descouvemont (1985) for more information. The occurrence of forbidden states can be simulated by an adequate choice of the potential. According to Buck et al. (1977), the potential must contain a number of bound states $n_{r}$, equal to the number of forbidden states. This prescription leads to deep potentials, since they involve additional (unphysical) bound states. The calculation of $n_{r}$ is in general not obvious, except for nucleus-nucleon systems.

In this simple model, the capture cross sections are deduced from integrals involving scattering functions $g_{L_{i} I}^{J_{i} \pi_{i}}(r)$ at energy $E$, and bound-state wave functions $g_{L_{f} I} \pi_{f}(r)$

$$
I_{i f}^{\lambda}(E)=\int g_{L_{i}}^{J_{i} \pi_{i}}(E, r) r^{\lambda} g_{L_{f}}^{J_{f} \pi_{f}}(r) d r .
$$

We refer to Bertulani (2003) for more detail.

The main advantage of the potential model is its simplicity. However, it assumes from the very beginning that the final bound state presents the two-body structure of the entrance channel. This is also true for resonances, which must be described by 
the adopted nucleus-nucleus structure. This hypothesis is not always valid. In the ${ }^{16} \mathrm{O}(\alpha, \gamma)^{20} \mathrm{Ne}$ reaction for example, the $0_{1}^{+}$ ground state and the $0_{4}^{+}$broad resonance are well described by an $\alpha+{ }^{16} \mathrm{O}$ structure, but the $0_{2}^{+}$and $0_{3}^{+}$resonances would require other configurations, such as $\alpha+{ }^{16} \mathrm{O}^{*}$ or $\mathrm{p}+{ }^{19} \mathrm{~F}$. This problem is more and more frequent as the level density increases. Another well-known example is the ${ }^{15} \mathrm{O}(\alpha, \gamma){ }^{19} \mathrm{Ne}$ reaction where most of the ${ }^{19} \mathrm{Ne}$ low-lying states can be accurately reproduced by an $\alpha+{ }^{15} \mathrm{O}$ structure, but where the resonance important for astrophysics $\left(3 / 2^{+}\right.$at $E_{c . m}$. $\left.=0.50 \mathrm{MeV}\right)$ most likely presents another structure (Dufour and Descouvemont, 2000).

\subsection{The Phenomenological R-Matrix Method}

The $R$-matrix method is well-known in atomic and nuclear physics (Descouvemont and Baye, 2010). The basic idea is to divide the space in two regions: the internal region (with radius a), where the nuclear force is important, and the external region, where the interaction between the nuclei is governed by the Coulomb force only. Although the $R$-matrix parameters do depend on the channel radius $a$, the sensitivity of the cross section with respect to its choice is quite weak. In the $R$-matrix method, the energy dependence of the cross sections is obtained from Coulomb functions, as expected from the Schrödinger equation.

In the phenomenological variant of the $R$-matrix method, the physics of the internal region is determined by a number $N$ of poles, which are characterized by their energies $E_{\lambda}$ and reduced widths $\gamma_{\lambda i}$. In a multichannel problem, the $R$-matrix at energy $E$ is defined as

$$
R_{i f}(E)=\sum_{\lambda=1}^{N} \frac{\gamma_{\lambda i} \gamma_{\lambda f}}{E_{\lambda}-E},
$$

which must be determined for each partial wave $J \pi$ (not written for the sake of clarity). Indices $i$ and $f$ refer to the initial and final channels. The pole properties are associated with the physical energy and width of resonances, but not strictly equal. This is known as the difference between "formal" and "observed" parameters, deduced from experiment.

The scattering matrices, and therefore the cross sections, are directly determined from the $R$ matrices in the different partial waves (see Lane and Thomas, 1958; Descouvemont and Baye, 2010 for detail). When a single-channel is involved $(i=f=1)$, the scattering matrix is written as

$$
U=\frac{I(k a)}{O(k a)} \frac{1-(S-i P) R}{1-(S+i P) R},
$$

where $P(E)$ and $S(E)$ are the penetration and shift factors, respectively. In that case, the $R$-matrix has a dimension $1 \times 1$. This definition can be easily extended to multichannel calculations (Descouvemont and Baye, 2010). The phase shift is defined by

$$
U=\exp (2 i \delta)=\exp \left(2 i\left(\delta_{\mathrm{HS}}+\delta_{\mathrm{R}}\right)\right),
$$

where $\delta_{H S}$ is the hard-sphere phase shift which is obtained with $R=0$, and therefore with $\gamma_{\lambda i}=0$. The hard-sphere and $R$-matrix phase shifts are obtained from

$$
\begin{aligned}
& \delta_{\mathrm{HS}}=-\arctan \frac{F(k a)}{G(k a)}, \\
& \delta_{\mathrm{R}}=\arctan \frac{P R}{1-S R} .
\end{aligned}
$$

Let us discuss the calculation of resonance properties. The pole energies $E_{\lambda}$ and reduced widths $\gamma_{\lambda}$ (see Equation 39) are associated with the poles of the $R$-matrix, and therefore depend on the conditions of the calculation, such as the radius $a$. In a single-channel problem, the resonance energies $E_{i}^{r}$, also referred to as the "observed" energies, are defined as the energies where the $R$-matrix phase shift is $\delta_{R}=\pi / 2$. According to (42), $E_{i}^{\mathrm{r}}$ are therefore solutions of the equation

$$
S\left(E_{i}^{\mathrm{r}}\right) R\left(E_{i}^{\mathrm{r}}\right)=1 .
$$

On the other hand, the "observed" width enters the Breit-Wigner parameterization near the resonance energy

$$
\delta_{\mathrm{R}}(E) \approx \arctan \frac{\Gamma_{i}(E)}{2\left(E_{i}^{\mathrm{r}}-E\right)},
$$

which gives, by using (42)

$$
\Gamma_{i}(E)=2 P(E) \frac{R\left(E_{i}^{\mathrm{r}}\right)}{[S(E) R(E)]_{E=E_{i}^{\mathrm{r}}}^{\prime}}=2 P(E) \tilde{\gamma}_{i}^{2},
$$

and defines $\tilde{\gamma}_{i}^{2}$ as the "observed" reduced width of the resonance. We also have

$$
\Gamma_{i}(E)=\Gamma_{i} \frac{P(E)}{P\left(E_{i}^{\mathrm{r}}\right)},
$$

where $\Gamma_{i}$ is the width calculated at the resonance energy.

If the pole number $N$ is larger than unity, or in multichannel calculations, the definition of $E_{i}^{\mathrm{r}}$ and of $\tilde{\gamma}_{i}^{2}$ is not analytical and requires numerical calculations (Angulo and Descouvemont, 2000; Brune, 2002). We illustrate here a simple but frequent situation of a single-channel calculation with $N=1$. The phase shift (42) is

$$
\delta_{\mathrm{R}}(E)=\arctan \frac{\Gamma_{1}(E)}{2\left(E_{1}-E-\gamma_{1}^{2} S(E)\right)},
$$

which is equivalent to (44) if we set

$$
\begin{aligned}
& E_{1}^{\mathrm{r}}=E_{1}-\tilde{\gamma}_{1}^{2} S\left(E_{1}^{\mathrm{r}}\right) \\
& \tilde{\gamma}_{1}^{2}=\gamma_{1}^{2} /\left(1+\gamma_{1}^{2} S^{\prime}\left(E_{1}^{\mathrm{r}}\right)\right),
\end{aligned}
$$

where $S^{\prime}(E)=d S / d E$. These formulas provide a simple link between calculated and observed values. To derive (48) we have used the Thomas approximation where the shift factor is linearized near $E_{1}^{\mathrm{r}}$ as

$$
S(E) \approx S\left(E_{1}^{\mathrm{r}}\right)+\left(E-E_{1}^{\mathrm{r}}\right) S^{\prime}\left(E_{1}^{\mathrm{r}}\right) .
$$


This approximation is in general quite accurate. The term $\tilde{\gamma}_{1}^{2} S^{\prime}\left(E_{1}^{\mathrm{r}}\right)$ is called the shift factor; it is proportional to the reduced width and is therefore large for strongly deformed states. With Equation (48), the fitting procedure can be used from the observed parameters. It presents important simplifications when some of the parameters (such as bound-state or resonance energies) are known from experiment, and therefore should be constant during the fit.

The phenomenological $R$-matrix method can be applied to transfer as well as to capture reactions. It is usually used to investigate resonant reactions but is also suited to describe non-resonant processes. In the latter case, the nonresonant behavior is simulated by a high-energy pole, referred to as the background contribution, which makes the $R$-matrix nearly energy independent. In nuclear astrophysics, a famous application is the ${ }^{12} \mathrm{C}(\alpha, \gamma){ }^{16} \mathrm{O}$ reaction. Experimental cross sections are available down to $E \approx 1 \mathrm{MeV}$, whereas the relevant stellar energy is close to $0.3 \mathrm{MeV}$. The $R$-matrix parameters are fitted to the available data, and then used to extrapolate the cross section to stellar energies (see a review in deBoer et al., 2017).

\subsection{Indirect Methods}

The main problem of nuclear astrophysics is the smallness of the cross sections at stellar energies. Further, in some reactions the availability of the required beam at the right energy may be difficult to obtain. These issues spurred interest in devising indirect methods (Tribble et al., 2014; Mukhamedzhanov and Rogachev, 2017), whereby the induced desired reaction is extracted from another reaction. We give below a brief overview of some indirect methods.

\subsubsection{The Trojan Horse Method}

The basic idea behind the Trojan Horse Method (Baur, 1986; Typel and Baur, 2003; Tumino et al., 2013; Spitaleri et al., 2019) is to use the three-body reaction,

$$
a+A \rightarrow b+(x+A) \rightarrow b+(c+C)
$$

to extract the cross section of the desired $x+A \rightarrow c+C$ two-body reaction. In the entrance channel, nucleus $a$ presents a cluster structure $a=b+x$, and cluster $x$ is transferred to the target $A$. In this process, cluster $b$ acts as a spectator.

Using the Plane Wave Impulse Approximation and the spectator model (Typel and Baur, 2003), the cross section of process (50) is factorized as

$$
\frac{d^{3} \sigma}{d E_{c} d \Omega_{c} d \Omega_{C}}=K\left(\frac{d \sigma}{d \Omega}\right)_{\mathrm{off}}\left|\Phi\left(\boldsymbol{k}_{x b}\right)\right|^{2},
$$

where $K$ is a kinematic factor. In this equation, $\left(\frac{d \sigma}{d \Omega}\right)_{\text {off }}$ is the half-off-energy-shell differential cross section for the two-body reaction, $x+A \rightarrow c+C$, and $\Phi\left(\boldsymbol{k}_{x b}\right)$ is the Fourier transform of the ground state wave function of nucleus $a(=b+x)$.

The main advantage of the Trojan Horse Method is that the cross section (51) is not affected by Coulomb effects, and is therefore more accessible than cross sections of astrophysical interest. Dividing it by a calculated $K\left|\Phi\left(\boldsymbol{k}_{x b}\right)\right|^{2}$ provides the desired cross section $\left(\frac{d \sigma}{d \Omega}\right)_{\text {off }}$. We refer the reader to Tribble et al. (2014) and Spitaleri et al. (2019) for recent reviews. The Trojan Horse Method has been applied to many reactions (see a review in Tumino et al., 2013). A recent example is the measurement of the ${ }^{2} \mathrm{H}(\mathrm{d}, \mathrm{p})^{3} \mathrm{H}$ and ${ }^{2} \mathrm{H}(\mathrm{d}, \mathrm{n})^{3} \mathrm{He}$ cross sections from $2 \mathrm{keV}$ to $1.5 \mathrm{MeV}$ (Tumino et al., 2011). An ${ }^{3} \mathrm{He}$ beam was used to measure the three-body ${ }^{2} \mathrm{H}\left({ }^{3} \mathrm{He}, \mathrm{p}^{3} \mathrm{H}\right){ }^{1} \mathrm{H}$ and ${ }^{2} \mathrm{H}\left({ }^{3} \mathrm{He}, \mathrm{n}^{3} \mathrm{He}\right){ }^{1} \mathrm{H}$ cross sections. Here nucleus $a$ is ${ }^{3} \mathrm{He}=\mathrm{d}+\mathrm{p}$, and the deuteron cluster ( $x$ in our notations) is transferred to the target. This approach provides ${ }^{2} \mathrm{H}(\mathrm{d}, \mathrm{p})^{3} \mathrm{H}$ and ${ }^{2} \mathrm{H}(\mathrm{d}, \mathrm{n})^{3} \mathrm{He}$ cross sections free of electron screening effects.

\subsubsection{Coulomb Dissociation}

The Coulomb breakup method (Baur et al., 1986, 2001) has been suggested in experiments using radioactive beams to address the problem of small cross sections. The photodissociation reaction

$$
c+\gamma \rightarrow a+b
$$

represents the reverse process of the capture reaction

$$
a+b \rightarrow c+\gamma
$$

and their cross sections $\sigma_{d}$ and $\sigma_{c}$ are related by the balance theorem

$$
\sigma_{d}(E)=\frac{\left(2 I_{a}+1\right)\left(2 I_{b}+1\right)}{2\left(2 I_{c}+1\right)} \frac{k^{2}}{k_{\gamma}^{2}} \sigma_{c}(E)
$$

where $I_{i}$ represents the spin of nucleus $i$ In most applications, the photon wavelength $k_{\gamma}$ is much larger than the particle wavelength $k$, which means that

$$
\frac{k^{2}}{k_{\gamma}^{2}} \gg 1
$$

and $\sigma_{d}$ is significantly larger than $\sigma_{c}$. This method is therefore a good way to compensate the smallness of capture cross sections at low energies. It is, however, limited to capture reactions toward the ground state of nucleus $c$.

The ${ }^{6} \mathrm{Li} \rightarrow \alpha+\mathrm{d}$ breakup reaction was used to assess the method (Kiener et al., 1991), and reinvestigated recently (Hammache et al., 2010). Coulomb breakup has also been used with radioactive beams, to investigate reactions, such as ${ }^{14} \mathrm{O} \rightarrow{ }^{13} \mathrm{~N}+\mathrm{p}$ (Motobayashi et al., 1991; Kiener et al., 1993) or ${ }^{8} \mathrm{~B} \rightarrow{ }^{7} \mathrm{Be}+\mathrm{p}$ (Motobayashi et al., 1994; Kikuchi et al., 1998; Schümann et al., 2006). The importance of the nuclear interaction on the Coulomb dissociation has been discussed, for example, in Kumar and Bonaccorso (2012).

\subsubsection{The Asymptotic Normalization Coefficient (ANC) Method}

At large distances, the radial wave function for bound states tends to a Whittaker function (Abramowitz and Stegun, 1972); we have

$$
g_{L}^{J \pi}(r) \underset{r \rightarrow \infty}{\longrightarrow} C_{L}^{J \pi} W_{-\eta, L+1 / 2}\left(2 k_{B} r\right)
$$


where $C_{L}^{J \pi}$ is the so-called "asymptotic normalization coefficient" (ANC). For weakly bound states, the exponential decrease is slow, and the main contribution to the electromagnetic matrix elements arises from large distances. The capture cross section is then essentially determined by the ANC. A typical example is the ${ }^{7} \mathrm{Be}(\mathrm{p}, \gamma)^{8} \mathrm{~B}$ reaction, where the ${ }^{8} \mathrm{~B}$ ground state is bound by $137 \mathrm{keV}$ only. Of course, this is true at very low energies only (typically $\leq 100 \mathrm{keV}$ ); for higher energies, the inner part of the wave function and, consequently, the nuclear interaction play a role.

When the external-capture approximation is valid, the capture cross section to a final state $f$ can be written as

$$
\sigma_{c}\left(E, J_{f} \pi_{f}\right) \approx\left|C_{L_{f}}^{J_{f} \pi_{f}}\right|^{2} \tilde{\sigma}_{c}\left(E, J_{f} \pi_{f}\right),
$$

where $\tilde{\sigma}_{f}\left(E, J_{f} \pi_{f}\right)$ is independent of the model; its energy dependence is given by the properties of the Coulomb functions (Baye and Brainis, 2000). The measurement of the ANC is based on transfer reactions where a nucleon of the projectile is transferred to the target. Energies must be large enough to ensure a peripheral process which is sensitive to the external part of the wave functions only. An example is the ${ }^{7} \mathrm{Be}\left({ }^{3} \mathrm{He}, \mathrm{d}\right){ }^{8} \mathrm{~B}$ reaction which has been used to determine the ANC of ${ }^{8}$ B (Mukhamedzhanov et al., 1995).

\section{APPLICATIONS}

\subsection{Applications of Microscopic Models}

The knowledge of the ${ }^{2} \mathrm{H}(\mathrm{d}, \gamma)^{4} \mathrm{He},{ }^{2} \mathrm{H}(\mathrm{d}, \mathrm{p})^{3} \mathrm{H}$, and ${ }^{2} \mathrm{H}(\mathrm{d}, \mathrm{n})^{3} \mathrm{He}$ cross sections at astrophysical energies is of great interest. Aside from the astrophysical interest, the ${ }^{2} \mathrm{H}(\mathrm{d}, \gamma)^{4} \mathrm{He}$ capture reaction is extremely important from the nuclear physics viewpoint because its cross section at low energies (below $0.3 \mathrm{MeV}$ ) is expected to be dominated by $D$-wave components in the $\alpha$ particle. Hence it should be very sensitive to the tensor force in the $N N$ interaction (Sabourov et al., 2004).

An ab initio model has been used to study the phase shifts of the $p+{ }^{3} \mathrm{He}$ (Arai et al., 2010) and $d+d, p+{ }^{3} \mathrm{H}, n+{ }^{3} \mathrm{He}$ (Arai et al., 2011) systems. For the two-body $N N$ interaction $V_{i j}$, two different realistic potentials are used: AV8' (Pudliner et al., 1997) and G3RS (Tamagaki, 1968), that consist of central, tensor, and spinorbit components. Because the main aim is to clarify the role of the tensor force, it is useful to compare results obtained with the realistic interactions with that of an effective $N N$ interaction that contains no tensor force. The MN central potential (Thompson et al., 1977) is adopted with the standard value for the admixture parameter $u=1$.

The ${ }^{2} \mathrm{H}(\mathrm{d}, \mathrm{p})^{3} \mathrm{H}$ and ${ }^{2} \mathrm{H}(\mathrm{d}, \mathrm{n})^{3} \mathrm{He}$ reactions play an important role in Big-Bang nucleosynthesis. As the observed $D / H$ ratio is currently known with $1.5 \%$ accuracy, a high precision is required for the reaction rates. The cross sections have been measured by several groups (Leonard et al., 2006; Tumino et al., 2014), but the extrapolations down to low energies are still uncertain. A compilation of the latest data has been undertaken to reduce the extrapolation uncertainties (Coc et al., 2015). The ${ }^{2} \mathrm{H}(\mathrm{d}, \mathrm{p})^{3} \mathrm{H}$ and ${ }^{2} \mathrm{H}(\mathrm{d}, \mathrm{n})^{3} \mathrm{He} S$ factors are presented in Figure 2. They mainly occur from the transitions of the $d+d^{5} S_{2}$ channel to the

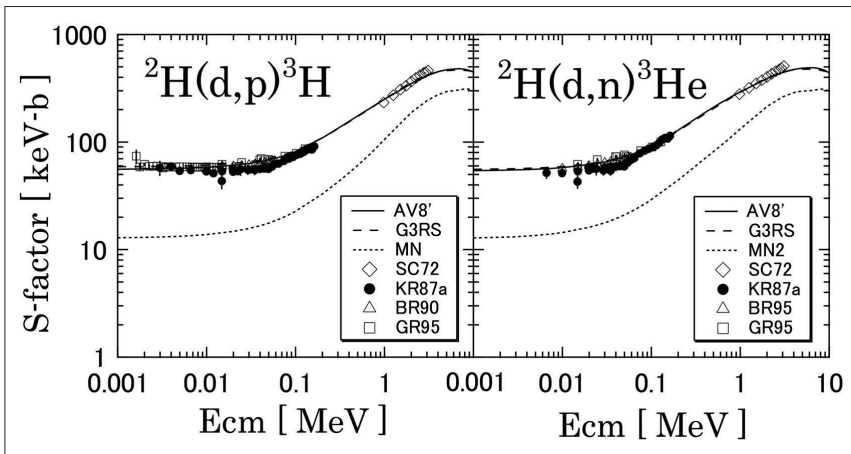

FIGURE $2 \mid{ }^{2} \mathrm{H}(\mathrm{d}, \mathrm{p})^{3} \mathrm{H}$ and ${ }^{2} \mathrm{H}(\mathrm{d}, \mathrm{n})^{3} \mathrm{He}$ astrophysical $S$-factors calculated with the realistic $A V 8^{\prime}+G 3 R S$ potential (solid lines) and with the effective MN potential (dotted lines). See Angulo et al. (1999) for the experimental data. Reprinted figure with permission from Arai et al. (2011). Copyright (2011) by the American Physical Society.

$D$-wave continuum of $p+{ }^{3} \mathrm{H}$ or $n+{ }^{3} \mathrm{He}$, which is due to the tensor force. Without the tensor force, these cross sections cannot be reproduced.

More recently, the ${ }^{3} \mathrm{He}(\alpha, \gamma)^{7} \mathrm{Be}$ and ${ }^{3} \mathrm{H}(\alpha, \gamma)^{7} \mathrm{Li}$ cross sections were computed in the no-core shell model (NCSM) (Dohet-Eraly et al., 2016). The authors used a renormalized chiral nucleon-nucleon interaction. In addition to NCSM states, which are optimized for bound states of the sevennucleon systems, a specific treatment of the $\alpha+{ }^{3} \mathrm{He}$ and $\alpha+{ }^{3} \mathrm{H}$ configuration is introduced, yielding the NCSM with continuum. With this correction, bound-state properties and elastic phase shifts are well-reproduced. Figure 3 shows the corresponding $S$-factors, which are compared with previous ab initio models (Nollett, 2001; Neff, 2011). Significant differences exist, due to convergence problems and to the use of different nucleonnucleon interactions.

\subsection{Applications of the Potential Model}

The first applications of the potential model were devoted to the ${ }^{3} \mathrm{He}(\alpha, \gamma){ }^{7} \mathrm{Be}$ (Tombrello and Parker, 1963; Buck et al., 1985) and the ${ }^{7} \mathrm{Be}(\mathrm{p}, \gamma)^{8} \mathrm{~B}$ (Robertson, 1973; Typel et al., 1997) reactions. Both reactions are essentially non-resonant at low energies, and the final bound states can be fairly well-described by a two-cluster structure. Most applications in the literature are performed with local potentials. More recently, the influence of the nonlocality was investigated by Tian et al. (2018). Applications to fusion reactions can be found, for example, in Chien et al. (2018) (single-channel model) and Assunção and Descouvemont (2013) (multichannel model).

We present in Figure 4 the ${ }^{3} \mathrm{He}(\alpha, \gamma)^{7} \mathrm{Be} S$-factor computed with the potential of Buck et al. (1985), and compare with some experimental data sets. The goal is not to provide a fit of the data, but rather to illustrate the use of the potential model in a simple example. The $\alpha+{ }^{3} \mathrm{He}$ potential contains a spin-orbit term; it reproduces several spectroscopic properties of ${ }^{7} \mathrm{Be}$ and of ${ }^{7} \mathrm{Li}$. We show separately the contributions of the $s$ wave $\left(L_{i}=0, J_{i}=1 / 2^{+}\right)$and of the $d$ wave $\left(L_{i}=2, J_{i}=\right.$ $\left.3 / 2^{+}, 5 / 2^{+}\right)$. Around $E=0$ the main contribution comes from 

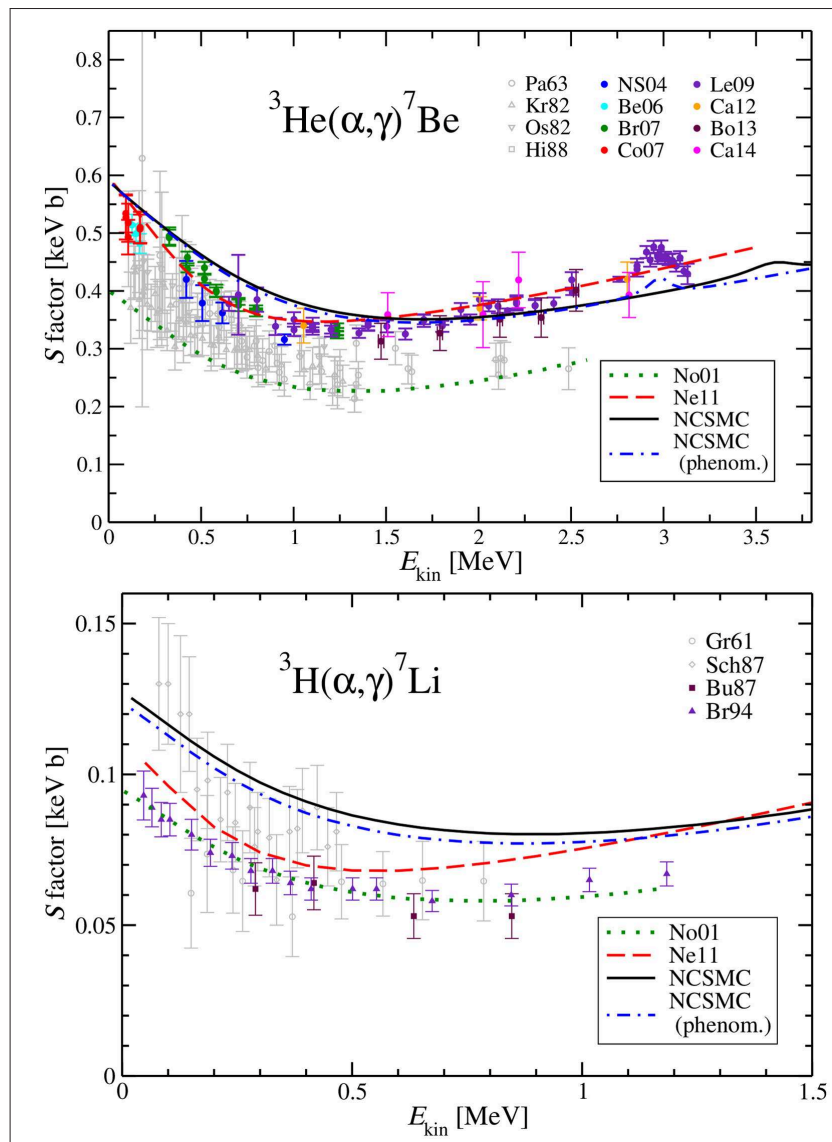

FIGURE $3 \mid{ }^{3} \mathrm{He}(\alpha, \gamma){ }^{7} \mathrm{Be}$ and ${ }^{3} \mathrm{H}(\alpha, \gamma)^{7} \mathrm{Li}$ S-factors computed with the No Core Shell model (solid lines). The other lines represent alternative calculations (see Dohet-Eraly et al., 2016 for details and for the references to the data). Reprinted from Dohet-Eraly et al. (2016) with permission from Elsevier.

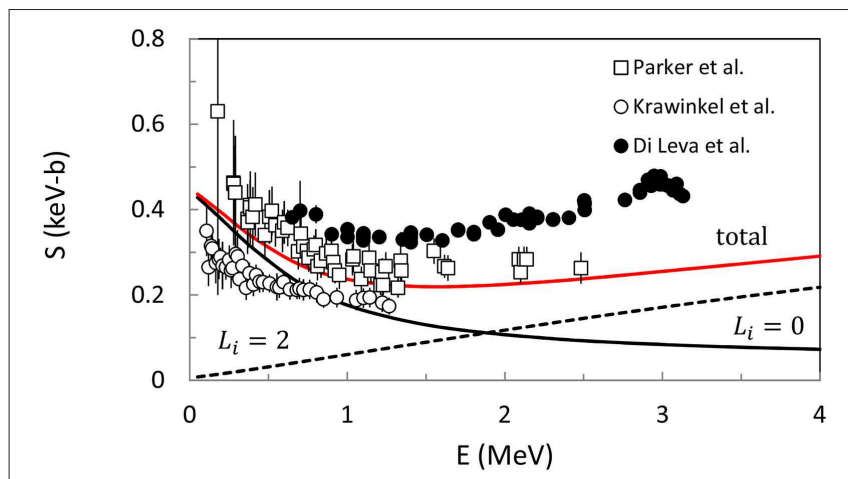

FIGURE $\left.4 \mid{ }^{3} \mathrm{He}(\alpha, \gamma)\right)^{7} \mathrm{Be} S$-factor computed in the potential model. The $L_{i}=0$ and $L_{i}=2$ components are shown separately. The data are taken from Parker and Kavanagh (1963), Kräwinkel et al. (1982), and Di Leva et al. (2009).

$L_{i}=0$, but $L_{i}=2$ cannot be neglected at energies where data are available.

As discussed in section 3, the capture cross sections are determined from integrals involving the initial and final wave

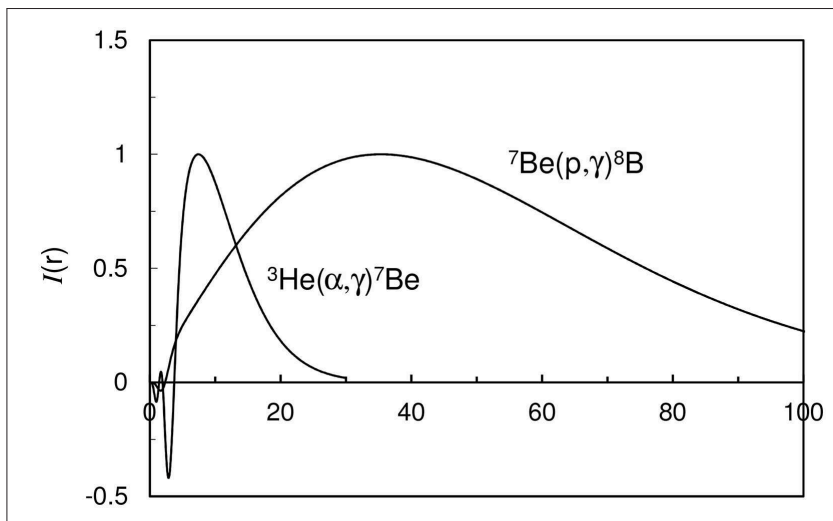

$r(f m)$

FIGURE 5 | Integrand (58) at $0.02 \mathrm{MeV}$ for ${ }^{7} \mathrm{Be}(\mathrm{p}, \gamma)^{8} \mathrm{~B}$, and at $0.1 \mathrm{MeV}$ for ${ }^{3} \mathrm{He}(\alpha, \gamma)^{7} \mathrm{Be}(\lambda=1)$. The normalization is arbitrary.

functions. Let us define

$$
I(r)=g^{J_{f}}(r) r^{\lambda} g^{J_{i}}(r),
$$

whose integral provides the electric component of the cross section. In Figure 5, we present this integrand for typical energies. For the ${ }^{3} \mathrm{He}(\alpha, \gamma)^{7} \mathrm{Be}$ reaction, the maximum of $I(r)$ is located near $r_{\max }=10 \mathrm{fm}$. At low energy the initial function $g^{J_{i}}(r)$ decreases rapidly in the nuclear region; conversely, the final function $g^{J_{f}}(r)$ is maximal in this region, and exponentially decreases as

$$
g^{I_{f}}(r)=C_{f} W_{-\eta, \ell_{f}+1 / 2}\left(2 k_{B} r\right) \sim C_{f} \exp \left(-k_{B} r\right) / \rho^{\eta},
$$

where $k_{B}$ is the wave number of the bound state. The decrease is therefore faster for large $k_{B}$ values, and hence for large binding energies. For very low binding energies, such as in ${ }^{8} \mathrm{~B}(-137 \mathrm{keV})$, we have $r_{\max } \approx 40 \mathrm{fm}$ and integrand (58) is non-negligible up to $150 \mathrm{fm}$. Accordingly, integrals (38) must be performed up to large $r$ values to ensure the convergence.

\subsection{Applications of the $\boldsymbol{R}$-Matrix Method}

Besides the typical application of the $R$-matrix to the ${ }^{12} \mathrm{C}(\alpha, \gamma){ }^{16} \mathrm{O}$ reaction (deBoer et al., 2017), various reactions have been analyzed recently in the $R$-matrix approach. Some examples are ${ }^{14} \mathrm{~N}(\mathrm{p}, \gamma)^{15} \mathrm{O}$ (Li et al., 2016) or ${ }^{13} \mathrm{C}(\mathrm{p}, \gamma)^{14} \mathrm{~N}$ (Chakraborty et al., 2015). Here we discuss in more detail a recent $R$-matrix analysis of the ${ }^{18} \mathrm{O}(\mathrm{p}, \alpha)^{15} \mathrm{~N}$ cross section which has been measured in the underground laboratory of the Gran Sasso (LUNA) (Bruno et al., 2019). This reaction influences the abundances of ${ }^{15} \mathrm{~N},{ }^{18} \mathrm{O}$ and ${ }^{19} \mathrm{~F}$ isotopes, critical to constrain a wide variety of stellar models.

At stellar temperatures, the rate is mainly determined by the properties of three $1 / 2^{+}(\ell=0)$ resonances at center of mass energies $0.143,0.610$, and $0.800 \mathrm{MeV}$. For the latter two resonances, results on their energy and partial widths are inconsistent (La Cognata et al., 2008), and important differences have also been reported between the cross sections of different 


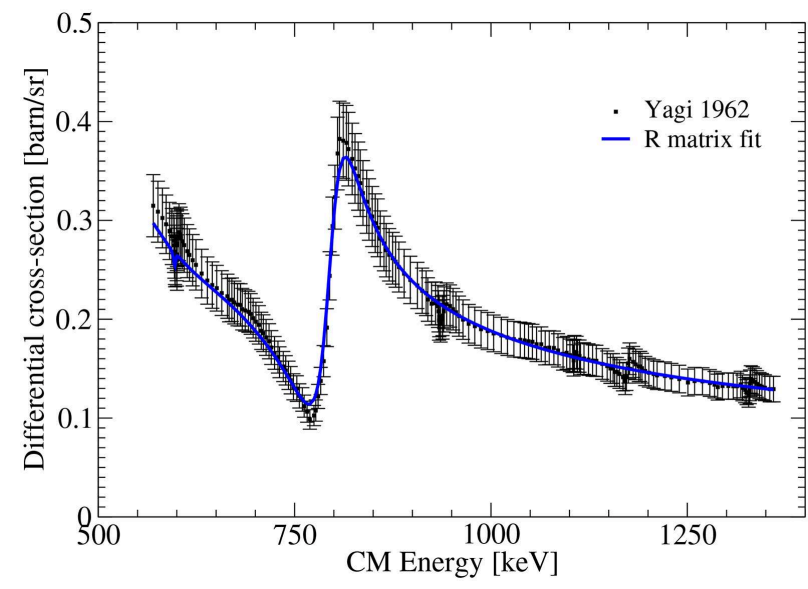

FIGURE $6 \mid{ }^{18} \mathrm{O}(\mathrm{p}, \mathrm{p})^{18} \mathrm{O}$ differential cross section at $\theta=140^{\circ}$ with the $R$-matrix fit. The data are taken from Yagi (1962). Reprinted from Bruno et al. (2019) with permission from Elsevier

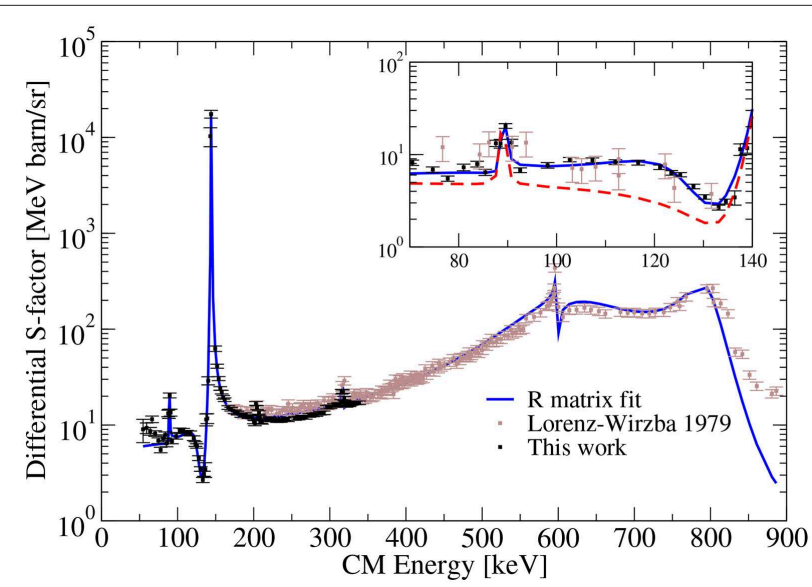

FIGURE $7 \mid{ }^{18} \mathrm{O}(\mathrm{p}, \alpha){ }^{15} \mathrm{~N}$ differential $S$-factor at $\theta=135^{\circ}$ with the $R$-matrix fit. The red dashed lined in the inset shows the effect of removing the new state at $E=106 \mathrm{keV}$. Reprinted from Bruno et al. (2019) with permission from Elsevier.

datasets at low energies. The main goal of Bruno et al. (2019) was to measure the non-resonant component of the cross section of the ${ }^{18} \mathrm{O}(\mathrm{p}, \alpha){ }^{15} \mathrm{~N}$ reaction at proton beam energies from $E_{p}=360$ to $60 \mathrm{keV}$, extending the range of direct measurements to stellar energies. From the cross section measurements, the strength of resonances of astrophysical interest could be determined.

An important advantage of the $R$-matrix theory is that some parameters are common to different reactions. In the present case, resonance energies, and proton widths are common to the ${ }^{18} \mathrm{O}(\mathrm{p}, \mathrm{p}){ }^{18} \mathrm{O}$ and ${ }^{18} \mathrm{O}(\mathrm{p}, \alpha){ }^{15} \mathrm{~N}$ cross sections. Only the $\alpha$ widths are specific to ${ }^{18} \mathrm{O}(\mathrm{p}, \alpha){ }^{15} \mathrm{~N}$. This property therefore provides several constraints on the parameter sets. Ideally, the cross sections should be measured at several angles, which permits to add further constraints.

The LUNA data and the fits of the ${ }^{18} \mathrm{O}(\mathrm{p}, \mathrm{p}){ }^{18} \mathrm{O}$ and ${ }^{18} \mathrm{O}(\mathrm{p}, \alpha){ }^{15} \mathrm{~N}$ cross sections are shown in Figures 6, 7, respectively. Several resonances are included in the fit (see Bruno et al., 2019 for detail). Both fits are excellent, with common parameters (energies and proton widths of resonances). The new data set (Bruno et al., 2019) suggest a new resonance at $E_{c m}=110 \mathrm{keV}$.

\section{CONCLUSION}

Nuclear astrophysics is a broad field, where many nuclear inputs are necessary. In particular, charged-particle cross sections are quite important, and difficult to measure, owing to the low energies and cross sections. Another characteristic of nuclear astrophysics is that there is almost no systematics. In the lowmass region, each reaction presents its own peculiarities and difficulties, in the theoretical as well as in the experimental viewpoints. Nevertheless some hierarchy can be established among reactions of astrophysical interest. Transfer reactions, arising from the nuclear interaction, present cross sections larger than capture cross sections which have an electromagnetic origin. In addition, the resonant or non-resonant nature of a reaction also affects the amplitude of the cross section.

We have discussed different theoretical models often used in nuclear astrophysics. The potential model and the $R$-matrix method are widely applied in this field; they are fairly simple and well-adapted to low-energy reactions. On the other hand, microscopic cluster models have a stronger predictive power, since they only rely on a nucleon-nucleon interaction, and on the assumption of a cluster structure for the nucleus. Finally, indirect methods are more and more developed since they overcome the major difficulty of nuclear astrophysics, i.e., the smallness of the cross sections.

A very impressive amount of work has been devoted to nuclear astrophysics in the last decades. Although most reactions involving light nuclei are sufficiently known, some reactions, such as ${ }^{12} \mathrm{C}(\alpha, \gamma){ }^{16} \mathrm{O}$ or ${ }^{12} \mathrm{C}+{ }^{12} \mathrm{C}$, still require much effort to reach the accuracy needed for stellar models. In the nucleosynthesis of heavy elements ( $s$ process, $p$ process), further problems arise from the level densities and the cross sections should be determined from statistical models. A better knowledge of these cross sections represents a challenge for the future.

\section{AUTHOR CONTRIBUTIONS}

The author confirms being the sole contributor of this work and has approved it for publication.

\section{FUNDING}

This work was supported by the Fonds de la Recherche Scientifique-FNRS under Grant Numbers 4.45.10.08 and J.0049.19. Computational resources have been provided by the Consortium des Équipements de Calcul Intensif (CÉCI), funded by the Fonds de la Recherche Scientifique de Belgique (F.R.S.-FNRS) under Grant No. 2.5020 .11 and by the Walloon Region. PD was Directeur de Recherches of F.R.S.-FNRS, Belgium. 


\section{REFERENCES}

Abramowitz, M., and Stegun, I. A. (1972). Handbook of Mathematical Functions. London: Dover.

Adelberger, E. G., Balantekin, A. B., Bemmerer, D., Bertulani, C. A., Chen, J. W., Costantini, H., et al. (2011). Solar fusion cross sections II: the pp chain and CNO cycles. Rev. Mod. Phys. 83:195. doi: 10.1103/RevModPhys.83.195

Afanasjev, A. V., Beard, M., Chugunov, A. I., Wiescher, M., and Yakovlev, D. G. (2012). Large collection of astrophysical $S$ factors and their compact representation. Phys. Rev. C 85:054615. doi: 10.1103/PhysRevC.85.054615

Alvarez-Ruso, L., Sajjad Athar, M., Barbaro, M. B., Cherdack, D., Christy, M. E., Coloma, P., et al. (2018). NuSTEC ${ }^{1}$ White Paper: Status and challenges of neutrino-nucleus scattering Prog. Part. Nucl. Phys. 100, 1-68. doi: 10.1016/j.ppnp.2018.01.006

Angulo, C., Arnould, M., Rayet, M., Descouvemont, P., Baye, D., Leclercq-Willain, C., et al. (1999). A compilation of charged-particle induced thermonuclear reaction rates. Nucl. Phys. A 656, 3-183. doi: 10.1016/S0375-9474(99)00030-5

Angulo, C., and Descouvemont, P. (2000). R-matrix analysis of interference effects in ${ }^{12} c(\alpha, \alpha){ }^{12} c$ and ${ }^{12} c(\alpha, \gamma){ }^{16}$ o. Phys. Rev. C 61:064611. doi: 10.1103/PhysRevC.61.064611

Aprahamian, A., Langanke, K., and Wiescher, M. (2005). Nuclear structure aspects in nuclear astrophysics. Prog. Part. Nucl. Phys. 54, 535-613. doi: 10.1016/j.ppnp.2004.09.002

Arai, K., Aoyama, S., and Suzuki, Y. (2010). Microscopic cluster model study of ${ }^{3} \mathrm{He}+p$ scattering. Phys. Rev. C 81:037301. doi: 10.1103/PhysRevC.81.037301

Arai, K., Aoyama, S., Suzuki, Y., Descouvemont, P., and Baye, D. (2011). Tensor force manifestations in $A b$ Initio study of the ${ }^{2} \mathrm{H}(d, \gamma)^{4} \mathrm{He}$, ${ }^{2} \mathrm{H}(d, p)^{3} \mathrm{H}$, and ${ }^{2} \mathrm{H}(d, n)^{3} \mathrm{He}$ reactions. Phys. Rev. Lett. 107:132502. doi: 10.1103/PhysRevLett.107.132502

Assunção, M., and Descouvemont, P. (2013). Role of the hoyle state in ${ }^{12} \mathrm{C}+{ }^{12} \mathrm{C}$ fusion. Phys. Lett. B 723, 355-359. doi: 10.1016/j.physletb.2013.05.030

Barker, F. C., and Kajino, T. (1991). The $12 \mathrm{C}(\alpha, \gamma) 160$ cross section at low energies. Aust. J. Phys. 44, 369-396. doi: 10.1071/PH910369

Baur, G. (1986). Breakup reactions as an indirect method to investigate low-energy charged-particle reactions relevant for nuclear astrophysics. Phys. Lett. B 178, 135-138. doi: 10.1016/0370-2693(86)91483-8

Baur, G., Bertulani, C. A., and Rebel, H. (1986). Coulomb dissociation as a source of information on radiative capture processes of astrophysical interest. Nucl. Phys. A 458, 188-204. doi: 10.1016/0375-9474(86)90290-3

Baur, G., Hencken, K., Trautmann, D., Typel, S., and Wolter, H. H. (2001). Electromagnetic dissociation as a tool for nuclear structure and astrophysics. Prog. Part. Nucl. Phys. 46, 99-108. doi: 10.1016/S0146-6410(01)0 0113-2

Baye, D. (1987). Supersymmetry between deep and shallow nucleus-nucleus potentials. Phys. Rev. Lett. 58, 2738-2741. doi: 10.1103/PhysRevLett.58.2738

Baye, D., and Brainis, E. (2000). Zero-energy determination of the astrophysical s factor and effective-range expansions. Phys. Rev. C 61:025801. doi: 10.1103/PhysRevC.61.025801

Baye, D., and Descouvemont, P. (1985). Antisymmetrization effects in radiative capture reactions. Ann. Phys. 165, 115-147. doi: 10.1016/S0003-4916(85)80007-5

Baye, D., and Kruglanski, M. (1992). $\alpha+\alpha$ scattering in a microscopic model with monopolar distortion. Phys. Rev. C 45, 1321-1332. doi: 10.1103/PhysRevC.45.1321

Bertulani, C. A. (2003). RADCAP: A potential model tool for direct capture reactions. Comput. Phys. Commun. 156, 123-141. doi: 10.1016/S0010-4655(03)00441-7

Bertulani, C. A., and Kajino, T. (2016). Frontiers in nuclear astrophysics. Prog. Part. Nucl. Phys. 89, 56-100. doi: 10.1016/j.ppnp.2016.04.001

Bethe, H. A. (1939). Energy production in stars. Phys. Rev. 55, 103. doi: $10.1007 / \mathrm{BF} 01325636$

Broggini, C., Bemmerer, D., Guglielmetti, A., and Menegazzo, R. (2010). LUNA: Nuclear astrophysics deep underground. Ann. Rev. Nucl. Part. Sci. 60, 53-73. doi: 10.1146/annurev.nucl.012809.104526

Brune, C. R. (2002). Alternative parametrization of $R$-matrix theory. Phys. Rev. C 66:044611. doi: 10.1103/PhysRevC.66.044611

Bruno, C. G., Aliotta, M., Descouvemont, P., Best, A., Davinson, T., Bemmerer, D., et al. (2019). Improved astrophysical rate for the ${ }^{18} \mathrm{O}(p, \alpha){ }^{15} \mathrm{~N}$ reaction by underground measurements. Phys. Lett. B 790, 237-242. doi: 10.1016/j.physletb.2019.01.017

Buck, B., Baldock, R. A., and Rubio, J. A. (1985). Cluster model of A = 7 nuclei and the astrophysical s factor for ${ }^{3} \mathrm{He}(\alpha, \gamma)^{7}$ Be at zero energy. J. Phys. G 11, L11-L16. doi: 10.1088/0305-4616/11/1/003

Buck, B., Friedrich, H., and Wheatley, C. (1977). Local potential models for the scattering of complex nuclei. Nucl. Phys. A 275, 246-268. doi: 10.1109/TNS.1977.4329048

Burbidge, E. M., Burbidge, G. R., Fowler, W. A., and Hoyle, F. (1957) Synthesis of the elements in stars. Rev. Mod. Phys. 29, 547-650. doi: 10.1103/RevModPhys.29.547

Canto, L. F., Gomes, P. R. S., Donangelo, R., and Hussein, M. S. (2006). Fusion and breakup of weakly bound nuclei. Phys. Rep. 424, 1-111. doi: 10.1016/j.physrep.2005.10.006

Canto, L. F., and Hussein, M. S. (2013). Scattering Theory of Molecules, Atoms and Nuclei. Singapore: World Scientific Publishing.

Caughlan, G. R., and Fowler, W. A. (1988). Thermonuclear reaction rates v. At. Data. Nucl. Data Tables 40, 283-334. doi: 10.1016/0092-640X(88)90009-5

Caurier, E., and Navrátil, P. (2006). Proton radii of ${ }^{4,6,8} \mathrm{He}$ isotopes from high-precision nucleon-nucleon interactions. Phys. Rev. C 73:021302. doi: 10.1103/PhysRevC.73.021302

Chakraborty, S., deBoer, R., Mukherjee, A., and Roy, S. (2015). Systematic Rmatrix analysis of the ${ }^{13} \mathrm{C}(p, \gamma){ }^{14} \mathrm{~N}$ capture reaction. Phys. Rev. $C$ 91:045801. doi: 10.1103/PhysRevC.91.049905

Chatterjee, R., Okolowicz, J., and Ploszajczak, M. (2006). Description of the ${ }^{17} \mathrm{~F}(p, \gamma){ }^{18} \mathrm{Ne}$ radiative capture reaction in the continuum shell model. Nucl. Phys. A 764, 528-550. doi: 10.1016/j.nuclphysa.2005.10.002

Chien, L. H., Khoa, D. T., Cuong, D. C., and Phuc, N. H. (2018). Consistent meanfield description of the ${ }^{12} \mathrm{C}+{ }^{12} \mathrm{C}$ optical potential at low energies and the astrophysical $s$ factor. Phys. Rev. C 98:064604. doi: 10.3952/physics.v57i4.3597

Clayton, D. D. (1983). Principles of Stellar Evolution and Nucleosynthesis. Chicago, IL: The University of Chicago Press.

Coc, A., Goriely, S., Xu, Y., Saimpert, M., and Vangioni, E. (2012). Standard big bang nucleosynthesis up to cno with an improved extended nuclear network. Astrophys. J. 744:158. doi: 10.1088/0004-637X/744/2/158

Coc, A., Petitjean, P., Uzan, J. P., Vangioni, E., Descouvemont, P., Iliadis, C., et al. (2015). New reaction rates for improved primordial D/H calculation and the cosmic evolution of deuterium. Phys. Rev. D 92:123526. doi: 10.1103/PhysRevD.92.123526

deBoer, R. J., Görres, J., Wiescher, M., Azuma, R. E., Best, A., Brune, C. R. et al. (2017). The ${ }^{12} \mathrm{C}(\alpha, \gamma){ }^{16} \mathrm{O}$ reaction and its implications for stellar helium burning. Rev. Mod. Phys. 89:035007. doi: 10.1103/RevModPhys.89.035007

Descouvemont, P. (1996). Application of an extended cluster model to the ${ }^{8} \mathrm{Li}(\alpha, \mathrm{n}){ }^{11} \mathrm{~B}$ reaction. Nucl. Phys. A 596, 285-298. doi: 10.1016/0375-9474(95)00409-2

Descouvemont, P. (2003). Theoretical Models for Nuclear Astrophysics. New York, NY: Nova Science.

Descouvemont, P., Adahchour, A., Angulo, C., Coc, A., and Vangioni-Flam, E. (2004). Compilation and $R$-matrix analysis of big bang nuclear reaction rates. At. Data Nucl. Data Tables 88, 203-236. doi: 10.1016/j.adt.2004.08.001

Descouvemont, P., and Baye, D. (1994). ${ }^{7} \mathrm{Li}+\mathrm{p}$ and ${ }^{7} \mathrm{Be}+\mathrm{n}$ reactions in a microscopic three-cluster model. Nucl. Phys. A 573, 28-46. doi: 10.1016/0375-9474(94)90013-2

Descouvemont, P., and Baye, D. (2010). The R-matrix theory. Rep. Prog. Phys. 73:036301. doi: 10.1088/0034-4885/73/3/036301

Descouvemont, P., and Dufour, M. (2012). Clusters in Nuclei, Vol. 2, Lecture Notes in Physics, Vol. 848. Berlin; Heidelberg: Springer.

Di Leva, A., Gialanella, L., Kunz, R., Rogalla, D., Schürmann, D., Strieder, F., et al. (2009). Stellar and primordial nucleosynthesis of ${ }^{7} \mathrm{be}$ : Measurement of ${ }^{3} h e(\alpha, \gamma)^{7}$ be. Phys. Rev. Lett. 102:232502. doi: 10.1103/PhysRevLett.102. 232502

Dohet-Eraly, J., Navrátil, P., Quaglioni, S., Horiuchi, W., Hupin, G., and Raimondi, F. (2016). ${ }^{3} \mathrm{He}(\alpha, \gamma){ }^{7} \mathrm{Be}$ and ${ }^{3} \mathrm{H}(\alpha, \gamma){ }^{7} \mathrm{Li}$ astrophysical $S$ factors from the no-core shell model with continuum. Phys. Lett. B 757, 430-436. doi: 10.1016/j.physletb.2016.04.021

Dufour, M., and Descouvemont, P. (2000). The ${ }^{15} \mathrm{~N}(\alpha, \gamma){ }^{19} \mathrm{~F}$ and ${ }^{15} \mathrm{O}(\alpha, \gamma){ }^{19} \mathrm{Ne}$ reactions in a microscopic multicluster model. Nucl. Phys. A 672, 153-164. doi: $10.1016 /$ S0375-9474(00)00054-3 
Dufour, M., and Descouvemont, P. (2011). Low-lying resonances in the 16b nucleus. Phys. Lett. B 696, 237-240. doi: 10.1016/j.physletb.2010.12.032

Friedrich, H. (1981). Microscopic nucleus-nucleus potentials. Phys. Rep. 74C, 209-275. doi: 10.1016/0370-1573(81)90131-9

Gaspard, D., Sparenberg, J. M., Wenda, Q., and Baye, D. (2019). Complexenergy analysis of proton-proton fusion. Phys. Rev. C 100:035805. doi: 10.1103/PhysRevC.100.035805

Hammache, F., Heil, M., Typel, S., Galaviz, D., Sümmerer, K., Coc, A., et al. (2010). High-energy breakup of ${ }^{6} \mathrm{Li}$ as a tool to study the big bang nucleosynthesis reaction ${ }^{2} \mathrm{H}(\alpha, \gamma){ }^{6}$ Li. Phys. Rev. C 82:065803. doi: 10.1103/PhysRevC.82.065803

Hofmann, H. M., and Hale, G. M. (1997). Microscopic calculation of the 4he system. Nucl. Phys. A 613, 69-106. doi: 10.1016/S0375-9474(96)00418-6

Horiuchi, H. (1977). Kernels of gcm, rgm and ocm and their calculation methods. Prog. Theor. Phys. Suppl. 62, 90-190. doi: 10.1143/PTPS.62.90

Horiuchi, H., Ikeda, K., and Katō, K. (2012). Recent developments in nuclear cluster physics. Prog. Theor. Phys. Suppl. 192:1. doi: 10.1143/PTPS.192.1

Hoyle, F. (1954). On nuclear reactions occuring in very hot stars.i. the synthesis of elements from carbon to nickel. Astrophys. J. Suppl. 1:121. doi: 10.1086/190005

Hussein, M. S. (1984). Theory of the heavy-ion fusion cross section. Phys. Rev. C 30:1962. doi: 10.1103/PhysRevC.30.1962

Iliadis, C. (2007). Nuclear Physics of Stars. Weinheim: Wiley-VCH Verlag GmbH.

José, J., and Iliadis, C. (2011). Nuclear astrophysics: the unfinished quest for the origin of the elements. Rep. Prog. Phys. 74:096901. doi: 10.1088/0034-4885/74/9/096901

Kamionkowski, M., and Bahcall, J. N. (1994). The rate of the proton-proton reaction. Astrophys. J. 420:884. doi: 10.1086/173612

Kiener, J., Gils, H. J., Rebel, H., Zagromski, S., Gsottschneider, G., Heide, N., et al. (1991). Measurements of the coulomb dissociation cross section of $156 \mathrm{mev}{ }^{6} \mathrm{Li}$ projectiles at extremely low relative fragment energies of astrophysical interest. Phys. Rev. C 44, 2195-2208. doi: 10.1103/PhysRevC.44.2195

Kiener, J., Lefebvre, A., Aguer, P., Bacri, C. O., Bimbot, R. B., Borderie, G. B., et al. (1993). Determination of the ${ }^{13} \mathrm{~N}(\mathrm{p}, \gamma){ }^{14} \mathrm{O}$ reaction rate through the coulomb break-up of a ${ }^{14} \mathrm{O}$ radioactive beam. Nucl. Phys. A 552, 66-81. doi: 10.1016/0375-9474(93)90331-Q

Kievsky, A., Rosati, S., Viviani, M., Marcucci, L. E., and Girlanda, L. (2008). A high-precision variational approach to three- and fournucleon bound and zero-energy scattering states. J. Phys. G 35:063101. doi: 10.1088/0954-3899/35/6/063101

Kikuchi, T., Motobayashi, T., Iwasa, N., Ando, Y., Kurokawa, M., Moriya, S., et al. (1998). Further measurement of the ${ }^{7} \mathrm{Be}(\mathrm{p}, \gamma)^{8} \mathrm{~B}$ cross section at low energies with the coulomb dissociation of ${ }^{8}$ B. Eur. Phys. J. A 3, 213-215.

Kräwinkel, H., Becker, H. W., Buchmann, L., Görres, J., Kettner, K. U., Kieser, W. E., et al. (1982). The ${ }^{3} \mathrm{He}(\alpha, \gamma)^{7} \mathrm{Be}$ reaction and the solar neutrino problem. Zeit. Phys. A 304, 307-332. doi: 10.1007/BF01421513

Kukulin, V. I., Neudatchin, V. G., Obukhovski, I. T., and Smirnov, Y. F. (1983). Clusters as Subsystems in Light Nuclei. Braunschweig: Vieweg.

Kumar, R., and Bonaccorso, A. (2012). Interplay of nuclear and coulomb effects in proton breakup from exotic nuclei. Phys. Rev. C 86:061601. doi: 10.1103/PhysRevC.86.061601

La Cognata, M., Spitaleri, C., Mukhamedzhanov, A. M., Irgaziev, B., Tribble, R. E., Banu, A., et al. (2008). Measurement of the 20 and $90 \mathrm{kev}$ resonances in the ${ }^{18} \mathrm{O}(\mathrm{p}, \alpha){ }^{15} \mathrm{~N}$ reaction via the trojan horse method. Phys. Rev. Lett. 101:152501. doi: 10.1103/PhysRevLett.101.152501

Lane, A. M., and Thomas, R. G. (1958). R-matrix theory of nuclear reactions. Rev. Mod. Phys. 30, 257-353. doi: 10.1103/RevModPhys.30.257

Leonard, D. S., Karwowski, H. J., Brune, C. R., Fisher, B. M., and Ludwig, E. J. (2006). Precision measurements of ${ }^{2} \mathrm{H}(d, p)^{3} \mathrm{H}$ and ${ }^{2} \mathrm{H}(d, n)^{3} \mathrm{He}$ total cross sections at big bang nucleosynthesis energies. Phys. Rev. C 73:045801. doi: 10.1103/PhysRevC.73.045801

Li, Q., Görres, J., deBoer, R. J., Imbriani, G., Best, A., Kontos, A., et al. (2016). Cross section measurement of ${ }^{14} \mathrm{~N}(p, \gamma)^{15} \mathrm{O}$ in the cno cycle. Phys. Rev. C 93:055806. doi: 10.1103/PhysRevC.93.055806

Liccardo, V., Malheiro, M., Hussein, M., Carlson, B., and Frederico, T. (2018). Nuclear processes in astrophysics: Recent progress. Eur. Phys. J. A 54:221. doi: 10.1140/epja/i2018-12648-5

Liu, Q. K. K., Kanada, H., and Tang, Y. C. (1981). Microscopic study of ${ }^{3} \mathrm{He}(\alpha, \gamma){ }^{7} \mathrm{Be}$ electric-dipole capture reaction. Phys. Rev. C 23, 645-656. doi: 10.1103/PhysRevC.23.645
Longland, R., Iliadis, C., Champagne, A., Newton, J., Ugalde, C., Coc, A., et al. (2010). Charged-particle thermonuclear reaction rates: I. monte carlo method and statistical distributions. Nucl. Phys. A 841, 1-30. doi: 10.1016/j.nuclphysa.2010.04.008

Marcucci, L. E. (2018). Recent progress in ab-initio studies of nuclear reactions of astrophysical interest with $\mathrm{a} \leq 3$. J. Phys. Conf. Series 981:012019. doi: 10.1088/1742-6596/981/1/012019

Marcucci, L. E., Schiavilla, R., and Viviani, M. (2013). Proton-proton weak capture in chiral effective field theory. Phys. Rev. Lett. 110:192503. doi: $10.1103 /$ PhysRevLett.110.192503

Motobayashi, T., Iwasa, N., Ando, Y., Kurokawa, M., Murakami, H., Gen, J. R., et al. (1994). Coulomb dissociation of ${ }^{8} \mathrm{~B}$ and the ${ }^{7} \mathrm{Be}(p, \gamma){ }^{8} \mathrm{~B}$ reaction at low energies. Phys. Rev. Lett. 73, 2680-2683. doi: 10.1103/PhysRevLett.73.2680

Motobayashi, T., Takei, T., Kox, S., Perrin, C., Merchez, F. K., Ieki, D. R., et al. (1991). Determination of the astrophysical ${ }^{13} \mathrm{~N}(p, \gamma){ }^{14} \mathrm{O}$ cross section through the coulomb dissociation method. Phys. Lett. B 264, 259-263. doi: 10.1016/0370-2693(91)90345-Q

Mukhamedzhanov, A. M., Gagliardi, C. A., and Tribble, R. E. (2001). Asymptotic normalization coefficients, spectroscopic factors, and direct radiative capture rates. Phys. Rev. C 63:024612. doi: 10.1103/PhysRevC.63.024612

Mukhamedzhanov, A. M., and Rogachev, G. V. (2017). Radiative capture reactions via indirect methods. Phys. Rev. C 96:045811. doi: 10.1103/PhysRevC.96.045811

Mukhamedzhanov, A. M., Tribble, R. E., and Timofeyuk, N. K. (1995). Possibility to determine the astrophysical $\mathrm{s}$ factor for the ${ }^{7} \mathrm{Be}(p, \gamma)^{8} \mathrm{~B}$ radiative capture from analysis of the ${ }^{7} \mathrm{Be}\left({ }^{3} \mathrm{He}, d\right)^{8} \mathrm{~B}$ reaction. Phys. Rev. C 51, 3472-3478. doi: 10.1103/PhysRevC.51.3472

Navrátil, P., and Quaglioni, S. (2011). Ab initio many-body calculations of deuteron $-{ }^{4} \mathrm{He}$ scattering and ${ }^{6} \mathrm{Li}$ states. Phys. Rev. C 83:044609. doi: 10.1103/PhysRevC.83.044609

Navrátil, P., and Quaglioni, S. (2012). Ab Initio many-body calculations of the ${ }^{3} \mathrm{H}(d, n){ }^{4} \mathrm{He}$ and ${ }^{3} \mathrm{He}(d, p){ }^{4} \mathrm{He}$ fusion reactions. Phys. Rev. Lett. 108:042503. doi: 10.1103/PhysRevLett.108.042503

Navrátil, P., Roth, R., and Quaglioni, S. (2010). Ab initio many-body calculations of nucleon scattering on ${ }^{4} \mathrm{He},{ }^{7} \mathrm{Li},{ }^{7} \mathrm{Be},{ }^{12} \mathrm{C}$, and ${ }^{16} \mathrm{O}$. Phys. Rev. C 82:034609. doi: 10.1103/PhysRevC. 82.034609

Neff, T. (2011). Microscopic calculation of the ${ }^{3} \mathrm{He}(\alpha, \gamma){ }^{7} \mathrm{Be}$ and ${ }^{3} \mathrm{H}(\alpha, \gamma)^{7} \mathrm{Li}$ capture cross sections using realistic interactions. Phys. Rev. Lett. 106:042502. doi: 10.1103/PhysRevLett.106.042502

Nollett, K. M. (2001). Radiative $\alpha$-capture cross sections from realistic nucleonnucleon interactions and variational monte carlo wave functions. Phys. Rev. C 63:054002. doi: 10.1103/PhysRevC.63.054002

Parker, P. D., and Kavanagh, R. W. (1963). $\mathrm{He}^{3}(\alpha, \gamma) \mathrm{Be}^{7}$ reaction. Phys. Rev. 131, 2578-2582. doi: 10.1103/PhysRev.131.2578

Pudliner, B. S., Pandharipande, V. R., Carlson, J., Pieper, S. C., and Wiringa, R. B. (1997). Quantum monte carlo calculations of nuclei with $a<$ 7. Phys. Rev. C 56:1720. doi: 10.1103/PhysRevC.56.1720

Rauscher, T., Thielemann, F. K., and Kratz, K. L. (1997). Nuclear level density and the determination of thermonuclear rates for astrophysics. Phys. Rev. C 56, 1613-1625. doi: 10.1103/PhysRevC.56.1613

Raynal, J. (1972). Computing as a Language of Physics, Trieste 1971. Vienna: IAEA. 281.

Reifarth, R., Erbacher, P., Fiebiger, S., Göbel, K., Heftrich, T., Heil, M., et al. (2018). Neutron-induced cross sections. Eur. Phys. J. Plus 133:424. doi: 10.1140/epjp/i2018-12295-3

Richter, W., Brown, B. A., Signoracci, A., and Wiescher, M. (2011). Shell-model studies of the rp reaction ${ }^{25} \mathrm{Al}(p, \gamma){ }^{26} \mathrm{Si}$. Prog. Part. Nucl. Phys. 66, 283-286. doi: 10.1016/j.ppnp.2011.01.021

Robertson, R. G. H. (1973). Proton capture by ${ }^{7}$ Be and the solar neutrino problem. Phys. Rev. C 7, 543-547. doi: 10.1103/PhysRevC.7.543

Rolfs, C., and Rodney, W. S. (1988). Cauldrons in the Cosmos. Chicago, IL: The University of Chicago Press.

Rose, H. J., and Brink, D. M. (1967). Angular distributions of gamma rays in terms of phase-defined reduced matrix elements. Rev. Mod. Phys. 39, 306-347. doi: 10.1103/RevModPhys.39.306

Sabourov, K., Ahmed, M. W., Canon, S. R., Crowley, B., Joshi, K., Kelley, J. H., et al. (2004). Experimental and theoretical study of the ${ }^{2} \mathrm{H}(\vec{d}, \gamma){ }^{4} \mathrm{He}$ reaction below $E_{\text {c.m. }}=60 \mathrm{keV}$. Phys. Rev. C 70:064601. doi: 10.1103/PhysRevC.70.064601 Satchler, G. R. (1983). Direct Nuclear Reactions. Oxford: Oxford University Press. 
Schümann, F., Typel, S., Hammache, F., Sümmerer, K., Uhlig, F., Böttcher, I., et al. (2006). Low-energy cross section of the ${ }^{7} \mathrm{Be}(p, \gamma)^{8} \mathrm{~B}$ solar fusion reaction from the coulomb dissociation of ${ }^{8} \mathrm{~B}$. Phys. Rev. C 73:015806. doi: 10.1103/PhysRevLett.73.015806

Spitaleri, C., La Cognata, M., Lamia, L., Pizzone, R. G., and Tumino, A. (2019). Astrophysics studies with the trojan horse method. Eur. Phys. J. A 55:161. doi: 10.1140/epja/i2019-12833-0

Tamagaki, R. (1968). Repulsive core of effective alpha-alpha potential and the pauli principle. Suppl. Prog. Theor. Phys. E68, 242-258. doi: 10.1143/PTPS.E68.242

Thielemann, F., Eichler, M., Panov, I., and Wehmeyer, B. (2017). Neutron star mergers and nucleosynthesis of heavy elements. Ann. Rev. Nucl. Part. Sci. 67, 253-274. doi: 10.1146/annurev-nucl-101916-123246

Thompson, D. R., LeMere, M., and Tang, Y. C. (1977). Systematic investigation of scattering problems with the resonating-group method. Nucl. Phys. A 286, 53-66. doi: 10.1016/0375-9474(77)90007-0

Thompson, I., and Nunes, F. (2009). Nuclear Reactions for Astrophysics: Principles, Calculation and Applications of Low-Energy Reactions. Cambridge: Cambridge University Press.

Thompson, I. J. (2010). NIST Handbook of Mathematical Functions. Cambridge: Cambridge University Press.

Tian, Y., Pang, D. Y., and Ma, Z. Y. (2018). Effects of nonlocality of nuclear potentials on direct capture reactions. Phys. Rev. C 97:064615. doi: 10.1103/PhysRevC.97.064615

Tombrello, T. A. (1965). The capture of protons by 7Be. Nucl. Phys. 71, 459-464. doi: 10.1016/0029-5582(65)90733-9

Tombrello, T. A., and Parker, P. D. (1963). Direct-capture model for the $\mathrm{He}^{3}(\alpha, \gamma) \mathrm{Be}^{7}$ and $\mathrm{T}(\alpha, \gamma) \mathrm{Li}^{7}$ reactions. Phys. Rev. 131, 2582-2589. doi: 10.1103/PhysRev.131.2582

Tribble, R. E., Bertulani, C. A., Cognata, M. L., Mukhamedzhanov, A. M., and Spitaleri, C. (2014). Indirect techniques in nuclear astrophysics: a review. Rep. Prog. Phys. 77:106901. doi: 10.1088/0034-4885/77/10/106901

Tumino, A., Spartá, R., Spitaleri, C., Mukhamedzhanov, A. M., Typel, S., Pizzone, R. G., et al. (2014). New determination of the ${ }^{2} \mathrm{H}(d, p)^{3} \mathrm{H}$ and ${ }^{2} \mathrm{H}(d, n)^{3} \mathrm{He}$ reaction rates at astrophysical energies. Astrophys. J. 785:96. doi: 10.1088/0004-637X/785/2/96

Tumino, A., Spitaleri, C., Cherubini, S., Gulino, M., La Cognata, M., Lamia, L., et al. (2013). New advances in the trojan horse method as an indirect approach to nuclear astrophysics. Few Body Syst. 54, 745-753. doi: 10.1007/s00601-013-0690-5

Tumino, A., Spitaleri, C., Mukhamedzhanov, A. M., Typel, S., Aliotta, M., Burjan, V., et al. (2011). Low-energy fusion reactions via the trojan horse method. Phys. Lett. B 700, 111-115. doi: 10.1016/j.physletb.2011. 05.001

Typel, S., and Baur, G. (2003). Theory of the trojan-horse method. Ann. Phys. 305, 228-265. doi: 10.1016/S0003-4916(03)00060-5

Typel, S., Wolter, H. H., and Baur, G. (1997). Higher-order effects in the coulomb dissociation of $8 \mathrm{~b}$ into ${ }^{7} \mathrm{Be}+\mathrm{P}$. Nucl. Phys. A 613, 147-164. doi: 10.1016/S0375-9474(96)0 0415-0

Volkov, A. B. (1965). Equilibrium deformation calculations of the ground state energies of $1 \mathrm{p}$ shell nuclei. Nucl. Phys. 74, 33-58. doi: 10.1016/0029-5582(65)90244-0

Wiescher, M., Käppeler, F., and Langanke, K. (2012). Critical reactions in contemporary nuclear astrophysics. Ann. Rev. Astron. Astrophys. 50, 165-210. doi: 10.1146/annurev-astro-081811-125543

Wildermuth, K., and Tang, Y. C. (1977). A Unified Theory of the Nucleus. Braunschweig: Vieweg.

Yagi, K. (1962). Analysis of elastic scattering of protons by 018 and the energy levels of f19. J. Phys. Soc. Japan 17:604. doi: 10.1143/JPSJ. 17.604

Zhang, X., Nollett, K. M., and Phillips, D. R. (2018). Models, measurements, and effective field theory: Proton capture on ${ }^{7} \mathrm{Be}$ at next-to-leading order. Phys. Rev. C 98:034616. doi: 10.3952/physics.v58i1.3647

Conflict of Interest: The author declares that the research was conducted in the absence of any commercial or financial relationships that could be construed as a potential conflict of interest.

Copyright (C) 2020 Descouvemont. This is an open-access article distributed under the terms of the Creative Commons Attribution License (CC BY). The use, distribution or reproduction in other forums is permitted, provided the original author(s) and the copyright owner(s) are credited and that the original publication in this journal is cited, in accordance with accepted academic practice. No use, distribution or reproduction is permitted which does not comply with these terms. 